\title{
Investigating the effects of hydrodynamics and mixing on mass transfer through the free-surface in stirred tank bioreactors
}

\author{
Anne de Lamotte ${ }^{\mathrm{a}, \mathrm{c}}$, Angélique Delafosse ${ }^{\mathrm{a}}$, Sébastien Calvo ${ }^{\mathrm{a}}$, Frank Delvigne ${ }^{\mathrm{b}}$, Dominique Toye ${ }^{\mathrm{a}}$ \\ ${ }^{a}$ Department of Chemical Engineering - Products, Environment, Processes (PEPs), University of Liège, Allée du 6 août 17B, 4000 Liège, Belgium \\ ${ }^{b}$ Gembloux Agro-Bio Tech - Microbial Processes E Interactions (MiPI), University of Liège, Passage des déportés 2, 5030 Gembloux, Belgium \\ ${ }^{c}$ National Funds for Scientific Research (FRS-FNRS), Rue d'Egmont 5, 1000 Brussels, Belgium
}

\begin{abstract}
In stirred-tank bioreactors, flow structures of various length and time scales are implied in scalar transport phenomena, such as gas species transfer through the liquid free-surface and their homogenization in the bulk. A proper understanding of the underlying mechanisms, i.e. hydrodynamics, mixing and mass transfer, and of their interactions is required to design and develop reliable and efficient production-scale bioprocesses. The objective of the present work is to experimentally investigate the coupling between gasliquid mass transfer of oxygen with mixing efficiency and circulation patterns inside an arbitrarily chosen stirred-tank configuration aerated through the liquid free-surface, a baffled 20L-vessel agitated by two Rushton turbines. Based on global parameter values, the most appropriate rotating speed, $N=300 \mathrm{rpm}$, is selected in order to further study local hydrodynamic quantities using Particle Image Velocimetry (PIV), as well as mixing and mass transfer dynamics using Planar Laser-Induced Fluorescence (PLIF). The results obtained with these local experimental methods are analyzed in detail. Their averages are first successfully compared to global data. Statistical analysis of their spatial distributions show that large-scale flow patterns significantly influence mass transfer through the free-surface of the stirred tank. Even if global measurements show that global characteristic times for mixing and mass transfer differ by two orders of magnitude, local experimental characterization shows persistent vertical gradients of dissolved gas concentrations. So the dissolved gas concentration is not as perfectly uniform as one might expect.
\end{abstract}

Keywords: Stirred tanks, Hydrodynamics, Mixing, Mass transfer, PIV, PLIF

\section{Introduction}

Stirred-tank reactors are commonly used in various chemical and biological processes because of their flexibility and good performance due to the wide range of available operating conditions. Besides, biological processes often require gasliquid contact for acceptable product formation. In some applications, aeration through the liquid free-surface is enough to meet the gas-liquid mass transfer demand. This is typically the case when either gas requirements/reactor volume are relatively low, or when bubbles are undesirable. Even when dealing with sparged reactors, aeration through the liquid free-surface is a factor to be considered when scaling up/down processes as its contribution to the overall mass transfer is linked to the evolution of the surface area to volume ratio (Godoy-Silva et al. (2009)).

In most stirred-tank reactors, hydrodynamics consists of turbulent flow structures of various length and time scales whose relative contributions to the interfacial transfer rate and to the mixing of scalar quantities such as dissolved gas concentration depend on the equipment-scale. Therefore, the design of a reliable and efficient industrial bioreactor requires a better identification of all characteristic scales relative to fluid dynamics, mixing and mass transfer. The dependence of these phenomena and of their interconnections regarding the equipment scale and geometry may be then properly understood. In practice, the influence of the vessel size on transport structures is usually not considered when scaling-up, leading then to suboptimal operations (Nienow (1997), Wernersson and Trägårdh (1999), Mathpati et al. (2009)). Traditional scale-up criteria based on the conservation of global quantities such as specific dissipated power $P / V$, mixing time $t_{\mathrm{M}}$ or overall mass transfer coefficient $K_{\mathrm{L}} a$ should be used with caution as they do not necessarily account for the heterogeneous spatial distributions of the flow variables and for their impact on process performance.

Consequently, the scale-up of a bioprocess from lab to industrial scale often results in a decrease of productivity compared to the lab-scale (Enfors et al. (2001)). Large gradients (substrate, nutrients, dissolved oxygen or carbon dioxide, $\mathrm{pH}$ ) appear when the bioreactor volume increases and can lead to modifications of the biological responses in terms of physiology or/and metabolism compared with lab-scale culture (Larsson et al. (1996), Delvigne et al. (2006), Garcia-Ochoa and Gomez (2009), Morchain et al. (2014)). Understanding and modeling these complex interactions between biological reaction and hydrodynamics are known to be key problems when dealing with bioprocesses, but can be expected only if hydrodynamics and physical (non biological) phenomena responsible for gradients are fully understood.

From fluid dynamics theory, it is well known that a turbulent flow can be divided into a macroscopic convection velocity field (large-scale motions, circulation) and a microscopic eddy-like 
velocity field which is, finally, dominated by diffusion (smallscale motions, turbulence). From this mechanistic representation, the mixing process is often divided into several simpler stages from the macro-scale to the micro-scale, i.e. from the scale of the whole vessel to finer scales associated to turbulent properties (Bałdyga and Pohorecki, 1995).

Gas-liquid mass transfer is also usually described by considering mechanisms occurring at these two scales (Martín et al., 2008). The exchange of species basically occurs through thin boundary layers at the gas-liquid interface by molecular diffusion processes. However, for poorly soluble gases, the transport of species is most often controlled by resistance on the liquid side which is dependent on flow structures and dynamics at and near the interface. Indeed, macro-scale motions determine the fluid paths across the reactor as well as their contribution to liquid free-surface aeration. They also lead to periodic and local deformation of the liquid free-surface. Besides, these largescale structures are directly affected by the reactor design, and thus also by its size. On the other hand, micro-scale motions are responsible for the rippled liquid free-surface and for the concentration gradients surrounding it and within the bulk. Turbulence is known to be of key importance for the gas transfer process. Many researchers have tried to explain the contribution of each flow scale on mass transfer process using conceptual models (e.g. Danckwerts (1951), Fortescue and Pearson (1967), Lamont and Scott (1970), McCready et al. (1986), Banerjee et al. (2004)). However, one of the main bottlenecks in the development and validation of detailed gas-liquid mass transfer models is the scarcity of reliable experimental information, relative to phenomena occurring near the interface. In parallel to the evolution of conceptual models, investigation techniques have thus also been improved. Even if computer simulations have provided estimates of required parameters for simple flow conditions at low turbulent Reynolds numbers, these simulations remain, up to now, too costly to be used for high Reynolds numbers.

Despite some research advances, the physical mechanisms of coupling between turbulence and gas-liquid mass transfer still remain unclear, such as the characteristic scales of eddies involved in the transfer or the effects of surface chemistry. Studies on mass transfer process across the air-water interface driven by grid-stirred turbulence provide some insights into the transfer mechanisms along with flow and scalar structures near the liquid free-surface (Chu and Jirka (1992), Herlina and Jirka (2004), Tsumori and Sugihara (2007), Janzen et al. (2010), Variano and Cowen (2013), Herlina and Wissink (2014)). These works indicate that the larger and smaller eddies are respectively able to distort and penetrate the boundary layer. In particular, surface-renewal motions observed in vertical planes have a much larger length-scale than the Kolmogorov scale $\eta$; they are rather within the range of the Taylor macro- and micro-scales, $\Lambda$ and $\lambda$.

The aim of the present work is thus to develop an experimental approach to characterize an arbitrarily chosen standard agitation configuration in terms of global and local quantities in order to better understand the coupling between gas-liquid mass transfer, mixing efficiency and circulation patterns.
For this purpose, a stirred-tank reactor aerated through the liquid free-surface is first characterized globally. The specific dissipated power, the mixing time and the global mass transfer coefficient are measured for different rotation speeds. The most appropriate agitation condition is selected on the basis of the values of these traditional scale-up criteria.

Then, local detailed information relative to a large volume fraction of the stirred vessel is collected to further study the system performance in terms of hydrodynamics, mixing and mass transfer. This requires accurate measurements of both flow quantities and concentration fields. To measure flow quantity field, Particle Image Velocimetry (PIV) is particularly appropriate, capable of measuring the flow field in an entire plane of the system with a few operations, yielding also enough information to obtain additional flow features such as turbulence and its characteristic length-scales (Bugay (1998), Escudié and Liné (2003), Khan et al. (2004), Micheletti et al. (2004), Gabriele et al. (2009)). To measure the concentration fields, Planar Laser-Induced Fluorescence (PLIF) has proven to be a suitable measurement technique. It has already been used to assess mixing characteristics (Houcine et al. (1996), Guillard et al. (2000), Fall et al. (2001), Busciglio et al. (2014)) in stirred-tank reactors, or to characterize mass transfer through an air-water interface, mainly using oxygen (Woodrow and Duke (2001), Herlina and Jirka (2004), Falkenroth et al. (2007), Janzen et al. (2010), Jimenez et al. (2013), Jimenez et al. (2014)) but also carbon dioxide (Tsumori and Sugihara (2007), Variano and Cowen (2013)). Besides, both techniques are considered as proper tools for numerical CFD model validation (Coroneo et al. (2011), Delafosse et al. (2014)). The results obtained with these local experimental methods are analyzed in detail. Their averages are compared to global data. Statistical analysis of their spatial distributions gives a further insight on the effects of hydrodynamics and mixing on mass transfer through the free-surface in stirred-tank bioreactors.

\section{Equipment and Methods}

\subsection{Stirred-tank configuration}

The investigated stirred-tank reactor, presented in Figure 1.a, has already been used in previous works (Delafosse et al. (2014)). This standard lab-scale set-up is a flat-bottom cylindrical vessel of diameter $T=0.22 \mathrm{~m}$, made in transparent Plexiglas, with four equally-spaced baffles. The centrally located mixing system consists of two four-blade Rushton turbines of diameter $D=0.1 \mathrm{~m}$ with a spacing $2 D$ and a clearance $D$ from the vessel bottom. The tank is filled with distilled water at $20^{\circ} \mathrm{C}$ (density $\rho_{\mathrm{L}}=998 \mathrm{~kg} \cdot \mathrm{m}^{-3}$, kinematic viscosity $\nu_{\mathrm{L}}=1.002 \cdot 10^{-6}$ $\mathrm{m}^{2} \cdot \mathrm{s}^{-1}$ ) up to a liquid height $2 T$, corresponding to a working volume $V=16.5 \mathrm{~L}$. To keep working liquid temperature constant, the tank is installed in a transparent Plexiglas rectangular container filled with tap water thermoregulated at $20^{\circ} \mathrm{C}$. Furthermore, that surrounding container minimizes optical distortion due to the curved tank wall during PIV and PLIF optical measurements. 
a)

b)
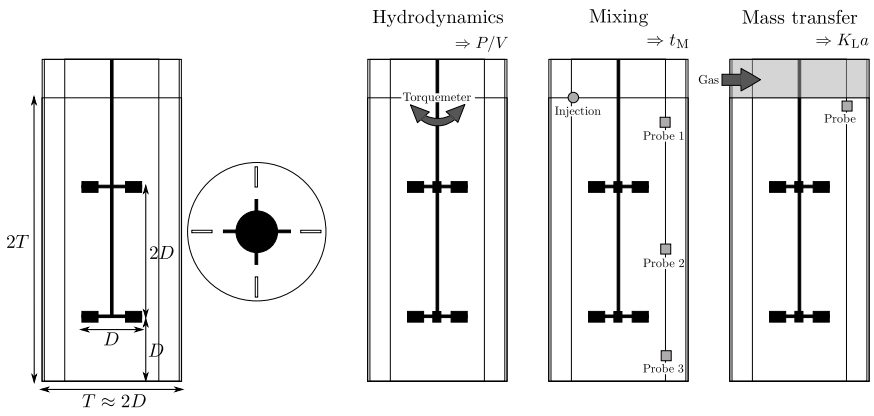

Figure 1: Stirred-tank configuration (a), Experimental set-up for global data acquisition methods (b)

\subsection{Global data acquisition methods}

In a first approach, global parameters (specific dissipated power $P / V$, mixing time $t_{\mathrm{M}}$ and mass transfer coefficient $K_{\mathrm{L}} a$ ) are used to quantify the system performance in terms of hydrodynamics, mixing and mass transfer. They are measured by means of accessible and simple experimental tools, illustrated in Figure 1.b: a torquemeter for $P / V$, a conductivity probe for $t_{\mathrm{M}}$ and a dissolved oxygen probe for $K_{\mathrm{L}} a$.

These macroscopic quantities are then analyzed to select the most appropriate agitation condition, i.e. the rotating speed $N \in$ [50 - 400] rpm, which will be subsequently used for the local characterization by means of the PIV and PLIF techniques.

\subsubsection{Hydrodynamics}

The global dissipated power $P / V$ of this mixing system is determined by measuring the mechanical torque $M$ exerted on the impeller shaft. For each rotating speed $N$, power losses associated to friction $M_{0}$ are subtracted from the measured power value. Information on energy consumption, in terms of power number $P_{0}$ and specific energy dissipation rate $\bar{\varepsilon}$, is then obtained:

$$
\frac{P}{V}=\frac{2 \pi N\left(M-M_{0}\right)}{V}=\rho_{\mathrm{L}} \bar{\varepsilon}=\frac{\rho_{\mathrm{L}} N^{3} D^{5} P_{0}}{V}
$$

\subsubsection{Mixing}

The global mixing time at $95 \%$ of homogeneity of an inert tracer $t_{\mathrm{M}}$ is determined using the conductivity technique. For each measurement, a pulse injection of a volume $V_{\mathrm{M}}=30 \mathrm{~mL}$ of sodium chloride saturated solution $\left([\mathrm{NaCl}]=359 \mathrm{~kg} \cdot \mathrm{m}^{-3}\right)$ is made at the liquid surface and the time evolution of sodium chloride concentration is then measured by means of conductivity probes. The influence of the tracer fluid properties on mixing time has been extensively investigated by, for instance, Burmester et al. (1992), Bouwmans et al. (1997), Gogate and Pandit (1999), Kasat and Pandit (2004)). Given these studies, the possible effects of the added liquid volume $\left(V_{M}=0.18 \%\right.$ of $V)$ and its density $\left(\rho_{\mathrm{M}}=1200 \mathrm{~kg} \cdot \mathrm{m}^{-3}\right)$ can be considered negligible for the present work.

Probes are placed at different locations in the reactor, i.e. at an angular position opposite to the injection point but at three different heights (just below the free surface, between the two impellers and at the bottom of the tank as depicted in Figure 1.b - Mixing). To obtain the global mixing time at $95 \% t_{\mathrm{M}}$ for the system, the three probe responses $C_{\mathrm{M}, i}(t)$ are normalized and combined using a root mean square of variance $\sigma_{\mathrm{M}, \mathrm{rms}}^{2}$ (Brown et al. (2004)):

$$
\begin{aligned}
t_{\mathrm{M}}= & t \text { for } \sigma_{\mathrm{M}, \mathrm{rms}}^{2}(t)=(0.05)^{2} \\
\text { with } \sigma_{\mathrm{M}, \mathrm{rms}}^{2}(t) & =\frac{1}{3} \sum_{i=1}^{3}\left(\frac{C_{\mathrm{M}, i}(t)-C_{\mathrm{M}, i}\left(t_{0}\right)}{C_{\mathrm{M}, i}\left(t_{\infty}\right)-C_{\mathrm{M}, i}\left(t_{0}\right)}-1\right)^{2}
\end{aligned}
$$

where $C_{\mathrm{M}, i}\left(t_{0}\right)$ and $C_{\mathrm{M}, i}\left(t_{\infty}\right)$ are the concentrations before tracer injection and after homogenization completion for each of the three probes.

\subsubsection{Mass transfer}

The global mass transfer coefficient $K_{\mathrm{L}} a$ is evaluated by the dynamic method of absorption and desorption.

The gas fed to the vessel is submitted to a step variation of concentration, i.e. an instantaneous change from either pure nitrogen or pure oxygen to air in order to perform either absorption or desorption experiments. The aeration is only made through the liquid free-surface by means of continuous flushing. Besides, the gas velocity is set low enough to avoid any interactions with the liquid free-surface but high enough to ensure that gas concentration remains constant in the headspace. Consequently, in the present study, mass transfer is not affected by the gas-phase behavior, contrary to the case of sparged or self-ingesting stirred-tank reactors (Linek et al. (1987), Scargiali et al. (2010)) where the uniformity of gas concentration in bubbles has to be ascertained. Besides, for low solubility gases such as oxygen, it is established that the resistance on the liquid side controls the transfer process $\left(1 / K_{\mathrm{L}} \simeq 1 / k_{\mathrm{L}}\right)$.

The dissolved oxygen concentration dynamics is followed from completely deaerated to air-saturated liquid using a dissolved $\mathrm{O}_{2}$ probe. The probe is placed just below the free-surface, and its time-dynamics (probe response time $<10 \mathrm{~s}$ ) is neglected as it is significantly smaller than the time for the oxygen transfer process (Van't Riet (1979)).

Given the assumption that the gas-side mass transfer resistance is negligible, the transport equation for dissolved oxygen concentration $C_{\mathrm{O}_{2}}$ is therefore:

$$
\frac{\mathrm{d} C_{\mathrm{O}_{2}}(t)}{\mathrm{d} t}=K_{\mathrm{L}} a\left(C_{\mathrm{O}_{2}}^{*}-C_{\mathrm{O}_{2}}(t)\right) \Rightarrow \ln \frac{C_{\mathrm{O}_{2}}^{*}-C_{\mathrm{O}_{2}}(t)}{C_{\mathrm{O}_{2}}^{*}-C_{\mathrm{O}_{2}}\left(t_{0}\right)}=-K_{\mathrm{L}} a \cdot t
$$

where $C_{\mathrm{O}_{2}}^{*}$ is the equilibrium concentration of dissolved oxygen with air.

\subsection{Local data acquisition methods}

The experimental tools for the measurements of global parameters are accessible and simple to set up. However, the use of these techniques to analyze mixing and mass transfer rests on probes which only give local values at specific locations. Besides, they can be considered, to some extent, as intrusive methods and may locally disturb the flow.

In order to fully understand the system performance in terms 
of hydrodynamics, mixing and mass transfer, the non-intrusive eulerian PIV and PLIF techniques are used to get local detailed information. Measurements are performed at the most relevant rotating speed selected on the basis of the macroscopic quantities $P / V, t_{\mathrm{M}}$ and $K_{\mathrm{L}} a$.

The experimental set-up for PIV and PLIF measurements consists of an optical equipment designed for vessel illumination and image detection, as illustrated in Figure 2. Measurements are performed on a vertical plane passing through the impeller axis and located at an angle of $\pi / 8$ behind one of the baffles. To visualize the flow over the entire height of the tank while maintaining a spatial resolution small enough to correctly estimate the turbulence quantities, PIV and PLIF measurements are performed simultaneously with two cameras: one for the bottom of the tank and the other one for the top as illustrated on Figure 2.

The light source is either a pulsed Nd:Yag laser (Litron, DualPower $65-15,2 \times 65 \mathrm{~mJ}$ at $15 \mathrm{~Hz}, 532 \mathrm{~nm}$ ) for PIV, or a continuous OPSL laser (Coherent, Genesis CX STM, 5 W, $532 \mathrm{~nm}$ ) for PLIF. Both lasers are equipped with a cylindrical lens for the light sheet generation. A continuous laser is chosen for PLIF measurements rather than a pulsed one, because of its superior beam quality and its stable continuous output that ensures the accuracy and reliability of the calibration of the system (Crimaldi, 2008). On the other hand, PIV measurements are less restrictive and only demand a high laser excitation intensity. Pulsed Nd:YAG lasers and their high power are then an obvious choice.

Two CCD cameras (Dantec Dynamics, Flowsense EO 4M-32, $2048 \times 2048$ pixel, $32 \mathrm{~Hz}$ ) are positioned orthogonally to the laser sheet and fitted with a $50 \mathrm{~mm}$ lens (Zeiss, Makro-Planar $\mathrm{T}^{*} 2 / 50 \mathrm{ZF}, 50 \mathrm{~mm}, \mathrm{f} / 2.0$ ). Both cameras are equipped by either a $550 \mathrm{~nm}$ high-pass filter for PIV or a $527-532 \mathrm{~nm}$ bandpass filter to only record the fluorescent light emitted by either particles for PIV or dye for PLIF.

The images are acquired through an imaging software platform (Dantec Dynamics, DynamicStudio 2015a) controlling a highresolution synchronization unit (Berkeley Nucleonics Corporation, Model 575 Pulse/Delay Generator). This unit triggers and stops simultaneously either both cameras and the pulsed laser for PIV, or both cameras only for PLIF.

\subsubsection{Hydrodynamics}

The liquid instantaneous radial and axial velocity fields, $U_{r}(r, z, t)$ and $U_{z}(r, z, t)$, are determined using the PIV technique. The liquid phase is seeded with inert fluorescent polyamide particles $\left(\rho_{\mathrm{P}}=1030 \mathrm{~kg} \cdot \mathrm{m}^{-3}\right)$ of mean diameter $d_{\mathrm{P}}$ $=5 \mu \mathrm{m}$. Their relaxation time $\tau_{\mathrm{P}} \approx 1.5 \mu \mathrm{s}$ (Eq. (4)) is smaller by several orders of magnitude than the Kolmogorov time scale $\tau_{\mathrm{K}} \in[950-28500] \mu \mathrm{s}$ (for $N \in[50-400] \mathrm{rpm}$ ) computed from the specific energy dissipation rate $\bar{\varepsilon}$ (Eq.5). Therefore, the seeding particles are good tracers of the turbulent flow and move with fluid elements.

$$
\tau_{\mathrm{P}}=\frac{\rho_{\mathrm{P}}}{\rho_{\mathrm{L}}} \frac{d_{\mathrm{P}}^{2}}{18 v_{\mathrm{L}}}
$$

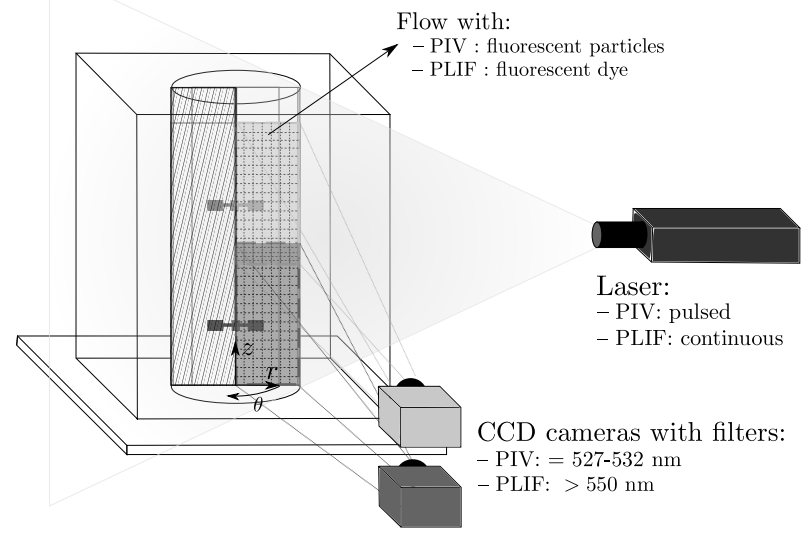

Figure 2: Experimental set-up for local data acquisition methods (PIV and PLIF)

$$
\tau_{\mathrm{K}}=\left(\frac{\nu_{\mathrm{L}}}{\bar{\varepsilon}}\right)^{\frac{1}{2}}
$$

The positions of the fluorescent particles in the plane illuminated by the laser sheet are recorded on image pairs at a frequency of $16 \mathrm{~Hz}$ by both cameras. The time between the two images of each pair is set to $125 \mu \mathrm{s}$. From each image pair, the velocity vectors are computed using the Adaptive-PIV technique which iteratively adjusts the size and shape of the individual interrogation areas from a maximum size of $64 \times 64$ pixel $^{2}$ to a minimum one of $8 \times 8$ pixel $^{2}$ with a grid step size of 4 , in order to adapt to local seeding densities and flow gradients (using Dantec software default settings). The resulting spatial resolution is $c a$. $0.6 \mathrm{~mm}$. The instantaneous vectors are then submitted to the default validation procedure combined with the Adaptive-PIV method, to prevent outliers from disturbing the iterations and thus the velocity measurements: first a peak validation on the image cross-correlation, and secondly a comparison of each vector to its neighbors using an outlier detection algorithm to detect false vectors. The number of image pairs is selected to assure statistical convergence of the velocity and turbulence quantities. Finally, a last validation based on the standard deviation is applied to each velocity component; all samples lying more than six times the standard deviation from the mean are rejected. The velocity fields are ensemble-averaged over 2175 (3 runs of 725) instantaneous measurements. The statistical convergence of the mean and fluctuating components of the velocity is reached at about 700 images.

\subsubsection{Mixing}

The inert tracer instantaneous concentration field $C_{\mathrm{M}}(r, z, t)$ is determined using the PLIF technique under the same operating conditions as conductivity experiments performed to assess the mixing time. The mixing dynamics within the vessel is followed by assessing the dispersion of a pulse injection of $30 \mathrm{~mL}$ of aqueous solution of Rhodamine- $6 \mathrm{G}$ ([Rh6G]= $8.25 \mathrm{~kg} \cdot \mathrm{m}^{-3} ; \mathrm{C}_{28} \mathrm{H}_{31} \mathrm{~N}_{2} \mathrm{O}_{3} \mathrm{Cl}$, CAS: 989-38-8, Sigma-Aldrich) rapidly injected just below the free-surface at an angular position opposite to the measurement plane.

The images are acquired at a frequency of $25 \mathrm{~Hz}$ and a spatial 
resolution of $c a .0 .12 \mathrm{~mm}$ by both cameras. Afterwards, these images are pre-processed using a pixel-by-pixel calibration procedure which converts the original intensity (grayscale value) images into concentration images according to the theory of fluorescence (Albani (2008), Lakowicz (2013)). The relationship between the fluorescent dye concentration $C_{\mathrm{M}}(r, z)$ and the intensity of emitted fluorescent light $I_{\mathrm{M}}(r, z)$ (pixel-wise mean of 50 frames) is simply linear and can be written for each pixel of an image as:

$$
I_{\mathrm{M}}(r, z)-I_{\mathrm{M}, 0}(r, z)=\kappa_{\mathrm{M}}(r, z) \cdot C_{\mathrm{M}}(r, z)
$$

where $I_{\mathrm{M}, 0}(r, z)$ is the measured fluorescence intensity in absence of the dye and $\kappa_{\mathrm{M}}(r, z)$ the proportionality constant which is a function of instrumental factors and system geometry.

\subsubsection{Mass transfer}

The dissolved oxygen instantaneous concentration field $C_{O_{2}}(r, z, t)$ is also determined using the PLIF technique. Measurements are performed under the same operating conditions as dynamic method experiments performed to assess the global mass transfer coefficient. The mass transfer dynamics at the liquid free-surface and within the vessel is followed on the basis of the time evolution of dissolved $\mathrm{O}_{2}$ concentration. For this purpose, the system is submitted to a step variation of oxygen concentration in the gas phase from pure oxygen to air in order to perform desorption experiments.

Even if absorption and desorption are mirror image processes under the conditions of the present study, PLIF measurements are only performed in the case of desorption because it has been show that it leads to somewhat more accurate results (Cents et al. (2005), Hamborg et al. (2010)).

An $\mathrm{O}_{2}$-sensitive fluorescent dye, a Ruthenium complex ( $\mathrm{C}_{36} \mathrm{H}_{24} \mathrm{Cl}_{2} \mathrm{~N}_{6} \mathrm{Ru} \cdot{ }_{x} \mathrm{H}_{2} \mathrm{O}$, CAS: 207802-45-7, Sigma-Aldrich; $\left.\left[\mathrm{Ru}^{\dagger}\right]=25 \mathrm{~kg} \cdot \mathrm{m}^{-3}\right)$, is added to the working liquid. This fluorophore, soluble in water, does not react and does not significantly alter the liquid phase properties (Jimenez et al. (2014)). Several sequences (at least five by experiment) of 1450 images are captured during the first two hours of these experiments, which is long enough to properly capture the exponential decay of dissolved oxygen concentration. The images are acquired by both cameras with a spatial resolution of $c a .0 .145 \mathrm{~mm}$ and at a frequency which varies depending on the acquisition sequence. Indeed, the acquisition frequency is $16 \mathrm{~Hz}$ at the beginning of the desorption process (the first two sequences) and then is decreased to 10, 5 and $1 \mathrm{~Hz}$ for the next sequences as the desorption process slows down. This procedure yields all the needed information relative to the dynamics of the phenomenon while sparing data treatment and storage. For example, the size of one instantaneous raw reconstructed data file, i.e. the combination of the intensity images from both cameras while masking pointless zones, is $c a$. 3.5 Mo. Besides, image buffer resources are anyway limited and then do not allow a continuous acquisition during a whole desorption experiment. Afterwards, these images are pre-processed using a pixel-by-pixel calibration procedure which converts the original intensity (grayscale value) images into concentration images according to the theory of fluorescence quenching (Albani (2008), Lakowicz (2013)). The fluorescent quencher concentration $C_{\mathrm{O}_{2}}(r, z)$ and the intensity of emitted fluorescent light $I_{\mathrm{O}_{2}}(r, z)$ (pixel-wise mean of 50 frames) are directly related through the Stern-Volmer equation and can be written for each pixel of an image as:

$$
\frac{I_{\mathrm{O}_{2}}(r, z)}{I_{\mathrm{O}_{2}, 0}(r, z)}=\frac{1}{1+\kappa_{\mathrm{O}_{2}}(r, z) \cdot C_{\mathrm{O}_{2}}(r, z)}
$$

where $I_{\mathrm{O}_{2}, 0}(r, z)$ is the measured fluorescence intensity in absence of oxygen and $\kappa_{\mathrm{O}_{2}}(r, z)$ is the Stern-Volmer constant.

\section{Results and Discussion}

Firstly, the macroscopic quantities (dissipated power $P / V$, mixing time $t_{\mathrm{M}}$ or overall mass transfer coefficient $K_{\mathrm{L}} a$ ) are analyzed to select the most appropriate agitation condition, i.e. the rotating speed $N \in[50-400] \mathrm{rpm}$, which is subsequently used for the local characterization by means of the PIV and PLIF techniques. These local experimental results are then presented and compared to global experimental data. Hydrodynamics and mixing outcomes provide respectively spatial and time information, which are used to refine the mass transfer dynamics investigation.

\subsection{Selection of the impeller rotating speed}

The selected rotating speed has to be the best compromise between mixing and mass transfer efficiency, on one hand, and power consumption, on the other hand. One also has to take technical constraints into account: PIV and PLIF measurements have to be performed at $N \leq 300 \mathrm{rpm}$ where it is low enough to avoid any gas ingestion and dispersion inside the reactor. At higher rotating speeds, air from the headspace begins to be incorporated into the liquid phase and an increasing number of circulating bubbles is observed. Laser light reflections on these bubbles induce noise which may significantly affect the quality of PIV and PLIF results.

The performance of the stirred-tank is first characterized in terms of global parameters related to these three phenomena: global dissipated power $P / V$, mixing time $t_{\mathrm{M}}$ and global mass transfer coefficient $K_{\mathrm{L}} a$. The results obtained for different operating conditions are reported in Figure 3 as a function of the logarithm of Reynolds number, $R e$ (Eq.(8)).

$$
R e=\frac{N D^{2}}{v_{\mathrm{L}}}
$$

As expected, the power measurements reveal a steep increase with the agitation speed. From these data, the power number is evaluated (Eq.(1)). It is equal to 5.965, which agrees with the value reported by Delafosse et al. (2014) for the same stirred-tank . Figure 3.a. shows that the fully turbulent regime is reached for $R e \geqslant 23$ 500, when $P_{0}$ becomes independent of $R e$. Therefore, in particular for scaling-up/down concerns, PIV and PLIF measurements have to be performed with $R e \geqslant 23500$ when turbulence controls the flow.

Figure 3.b. presents the evolution of the global mixing time 

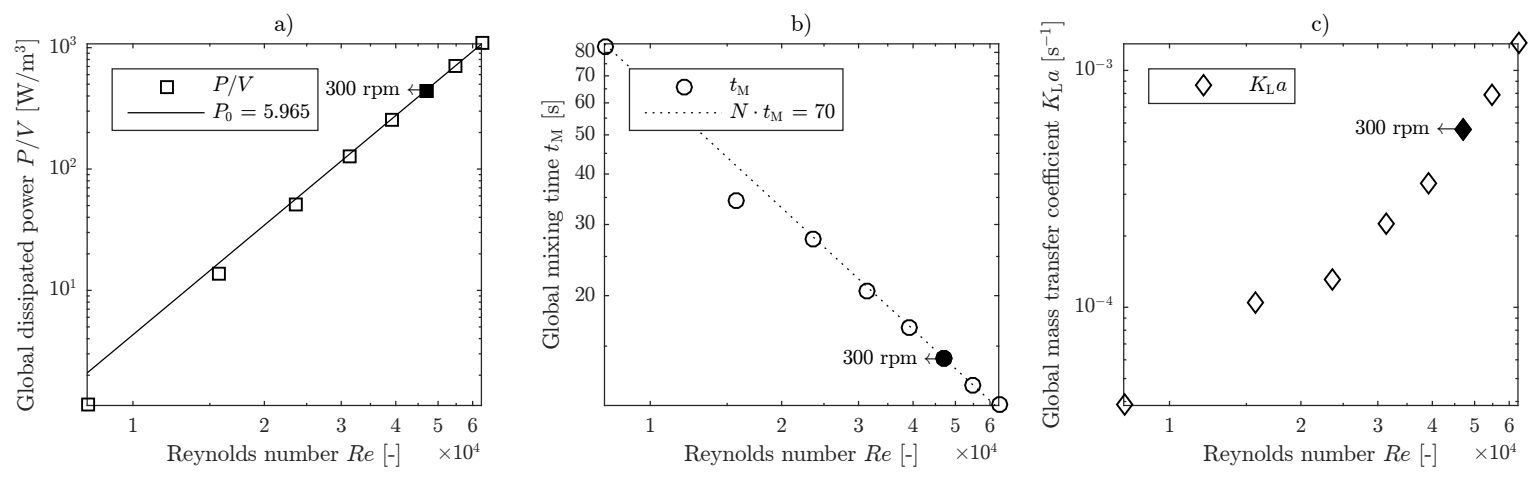

Figure 3: Global dissipated power (a), global mixing time (b) and global mass transfer coefficient (c) under different rotating speeds

as a function of the Reynolds number. It shows that, at high Reynolds numbers, the dimensionless number $N \cdot t_{\mathrm{M}}$ remains constant, equal to 70 in the present work.

Figure 3.c. shows that the global mass transfer coefficient increases with the rotating speed. While $N$ increases, the freesurface becomes wavier and its area $a$ increases. Due to higher mixing efficiency leading to a faster renewal rate of gas-liquid interface, the mass transfer coefficient $K_{\mathrm{L}}$ is expected to also increase. It results consequently in higher $K_{\mathrm{L}} a$ values.

From all theses results, it has been decided to perform all PIV and PLIF experiments at $N=300 \mathrm{rpm}(R e=47125)$ for which the flow is fully turbulent in the tank and which corresponds to a satisfactory compromise between a short mixing time, $t_{\mathrm{M}}=14$ $\mathrm{s}$, a good mass transfer performance, $K_{\mathrm{L}} a=4.4 \cdot 10^{-4} \mathrm{~s}^{-1}$, while limiting the power consumption, $P / V=448 \mathrm{~W} \cdot \mathrm{m}^{-3}$. This choice of velocity also allows meeting the technical constraint related to optical measurements as it does not induce any air entrainment from the liquid free-surface.

\subsection{Hydrodynamics}

At $N=300 \mathrm{rpm}$ the flow inside the stirred-tank is turbulent and is then fully three-dimensional. Admittedly, analysis based on 2D-PIV may be thus subject to an error as the tangential flow $U_{\theta}$ is not measured and therefore not properly taken into account. However, 2D measurements in a vertical plane in fully-baffled stirred-tank s may be used for a reasonably accurate estimate of the energy distribution and its dissipation in the 3D flow field while watching out that the spatial resolution of PIV system is fine enough (Chung et al. (2007), Liné et al. (2013)).

Following the Reynolds decomposition, the 2D-PIV instantaneous radial and axial velocity fields, $U_{r}(r, z, t)$ and $U_{z}(r, z, t)$ can be decomposed into a time-averaged mean component $\bar{U}(r, z)$ and a fluctuating component $u^{\prime}(r, z, t)$ :

$$
\begin{aligned}
& U_{r}=\overline{U_{r}}+u_{r}^{\prime} \\
& U_{z}=\overline{U_{z}}+u_{z}^{\prime}
\end{aligned}
$$

Figure 4.a presents the magnitude of the velocity vectors

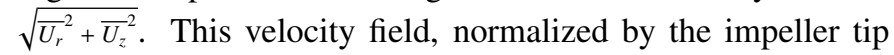
speed $N D$, reveals the typical flow pattern observed for the dual impeller Rushton turbine configuration: a stream is radially discharged from each turbine with a maximum velocity magnitude of $c a$. 1.8.ND. These flow streams are then swept up and down thanks to the baffles to form two large circulation loops in the vessel.

As the mean of the fluctuating components is by definition zero, it is normal practice to characterize them via their root mean square, $\sqrt{\overline{u_{r}^{\prime 2}}}(r, z)$ and $\sqrt{\overline{u_{z}^{\prime 2}}}(r, z)$. Figures 4.b and 4.c present the radial $\sqrt{\overline{u_{r}^{\prime 2}}}$ and axial $\sqrt{u_{z}^{\prime 2}}$ r.m.s. turbulent velocity components. The fields shown are normalized by the impeller tip speed, $N D$. The regions of highest r.m.s. fluctuating velocity fields for the axial and radial terms are in the impellers discharge. It is worth noting that PIV results may undergo some local inaccuracies because of the laser reflection on the free-surface and on the baffle located just behind the measurement plane. The two r.m.s. components differ in certain zones, indicating that turbulence is not locally isotropic. For instance, turbulence seems isotropic in the region of the liquid jet axis but anisotropic in several regions really close to the impeller (close to the disc and the impeller tip) or the axis. These observations agree with results from experimental studies of anisotropy of the flow field inside tanks stirred by a Rushton turbine (Derksen et al. (1999), Galletti et al. (2004), Escudié and Liné (2006)). The comparison with their results, obtained with only one impeller may be justified by the fact that, in the present work, the agitation configuration leads to two nearly parallel flow patterns, i.e. with no strong interaction between both impellers.

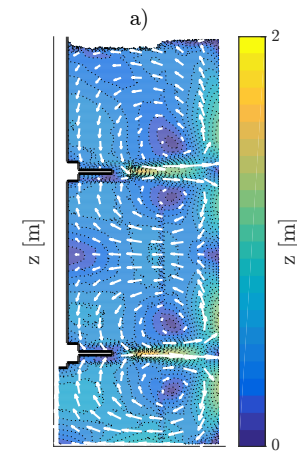

$\mathrm{r}[\mathrm{m}]$

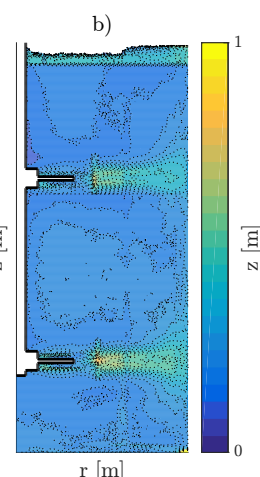

$\mathrm{r}[\mathrm{m}]$

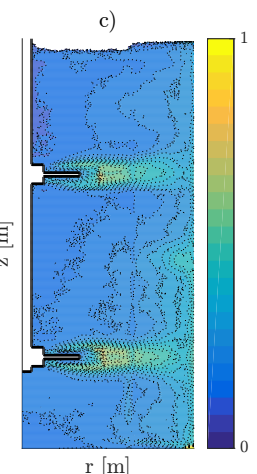

$\mathrm{r}[\mathrm{m}]$
Figure 4: The dimensionless magnitude of the mean velocity vectors $\sqrt{{\overline{U_{r}}}^{2}+{\overline{U_{z}}}^{2}}$ on the measurement plane (a), and the dimensionless r.m.s. turbulent velocity components on the measurement plane, $\sqrt{\overline{{u_{r}^{\prime}}^{2}}}(\mathrm{~b})$ and $\sqrt{\overline{u_{z}^{\prime 2}}}(\mathrm{c})$ 
The PIV technique is effective for detecting and quantifying the characteristic scales of turbulent eddies which play an important role in the mass transfer process as they drive surfacerenewal motions near the liquid free-surface. The continuous range of length-scales of a turbulent flow in a stirred-tank extends from the tank diameter $T$, the largest dimension, over at least three orders of magnitude to the Kolmogorov scale $\eta$ where the turbulent kinetic energy is dissipated as heat by molecular viscosity.

It is possible to estimate spatial distributions of only two characteristic length scales from the PIV instantaneous velocity fields: the Taylor macro (or integral) length-scale $\Lambda$ and the Taylor micro length-scale $\lambda$. Indeed, to accurately deduce the spatial distribution of $\eta$, the PIV spatial resolution should be equal or smaller than $\eta$. The mean Kolmogorov scale over the whole vessel $\bar{\eta}$ can be estimated from the global dissipation rate, $\bar{\varepsilon}=0.45 \mathrm{~m}^{2} \cdot \mathrm{s}^{-3}$, using Equation (10) and is found to be $c a .40$ $\mu \mathrm{m}$. The spatial resolution under the present experimental conditions thus corresponds to $c a$. 15 times the mean Kolmogorov scale; the PIV data are definitely filtering the smallest scales of turbulence.

$$
\bar{\eta}=\left(\frac{v^{3}}{\bar{\varepsilon}}\right)^{\frac{1}{4}}
$$

The Taylor macro-scale corresponds to the size of the most energetic structures and is generally assumed to be equivalent to the tenth of the impeller diameter or half the blade height. However, these large scales are generally not statistically isotropic, since they are determined by the particular geometrical features of the flow and its boundaries. The Taylor micro-scale is an intermediate one between the largest and smallest turbulent scales, and corresponds to the minimum size of eddies that significantly contributes to the turbulent kinetic energy. These small eddies are less dependent on the agitation system and tend to be more isotropic. Its comparison with the PIV spatial resolution is thus highly pertinent to assess the validity of the results of Figure 4 (Liné, 2016).

These two turbulent scales can be determined from correlation functions as proposed by Taylor (Taylor, 1935). Spatial autocorrelation functions of the velocity are determined from the second order moment of velocity fluctuations:

$$
\begin{aligned}
& R_{r r}(r+d r, z)=\frac{\overline{u_{r}^{\prime}(r, z, t) \cdot u_{r}^{\prime}(r+d r, z, t)}}{\sqrt{\overline{u_{r}^{\prime 2}(r, z, t)} \cdot \overline{u_{r}^{\prime 2}(r+d r, z, t)}}} \\
& R_{z z}(r, z+d z)=\frac{\overline{u_{z}^{\prime}(r, z, t) \cdot u_{z}^{\prime}(r, z+d z, t)}}{\sqrt{\overline{u_{z}^{\prime 2}(r, z, t)} \cdot \overline{u_{z}^{\prime 2}(r, z+d z t)}}}
\end{aligned}
$$

where $d r$ and $d z$ are the radial and axial space lags. The present study is only considering the longitudinal autocorrelations in which the direction of the spatial shift is parallel to the one of the velocity fluctuations $\left(u_{r}^{\prime} \| d r\right.$ and $u_{z}^{\prime} \| d z$ ), as the associated integral length-scales correspond to the characteristic lengths of the larger eddies (Hinze (1959), Jakobsen (2008)). The analysis of the evolution of these functions yields the two length-scales
$\Lambda$ and $\lambda$ :

$$
\begin{aligned}
& \Lambda_{r r}(r, z)=\int_{0}^{\infty} R_{r r}(r+d r, z) \cdot d r \\
& \Lambda_{z z}(r, z)=\int_{0}^{\infty} R_{z z}(r, z+d z) \cdot d z \\
& \lambda_{r r}(r, z)=\sqrt{\frac{-2}{\partial_{r=0}^{2} R_{r r}(r+d r, z)}} \\
& \lambda_{z z}(r, z)=\sqrt{\frac{-2}{\partial_{z=0}^{2} R_{z z}(r, z+d z)}}
\end{aligned}
$$

More details on the determination of the two characteristic length-scales from these equations are given in the Appendix.

Figures 5.a and 5.b shows the integral length-scales $\Lambda_{r r}$ and $\Lambda_{z z}$ maps, while their distributions within the measurement plane are displayed in Figure 5.c. Both mean macro-lengths, $\overline{\Lambda_{r r}}=0.011 \mathrm{~m}$ and $\overline{\Lambda_{z z}}=0.0127 \mathrm{~m}$, are nearly equivalent to the often-used value of half of the blade height $(w / 2=0.01 \mathrm{~m})$ which is the characteristic value of the radial jet that generates the macro-turbulent eddies. As expected from the anisotropy and inhomogeneity of the flow, $\Lambda_{r r}$ and $\Lambda_{z z}$ reveal different spatial patterns. The macro length-scale does not present much variation in the radial direction, but varies more significantly in the axial direction. Their distributions indicate that the integral length scales obtained for $u_{z}^{\prime}\left(\Lambda_{z z} \in\left[1.5 \cdot 10^{-3}, 3.5 \cdot 10^{-2}\right]\right.$ $\mathrm{m})$ are greater than those for $u_{r}^{\prime}\left(\Lambda_{r r} \in\left[1.5 \cdot 10^{-3}, 2.25 \cdot 10^{-2}\right]\right.$ $\mathrm{m})$. The highest values of $\Lambda_{z z}$ are located near the liquid freesurface and the turbines, while $\Lambda_{r r}$ appears more constant. For instance, the region located between the free-surface $(z=0.44$ $\mathrm{m})$ and above the upper impeller $(z \simeq 0.35 \mathrm{~m})$ is characterized by $\Lambda_{z z} \in[0.03,0.035] \mathrm{m}$. These results are coherent with the hydrodynamics associated to the agitation configuration, i.e. the (axial) influence of the baffles on the (radial) flow generated by the Rushton turbines.

Figures 6.a and 6.b present the Taylor micro-scales $\lambda_{r r}$ (a) and $\lambda_{z z}$ (b) maps, while their distributions within the measurement plane are shown in Figure 6.c. Spatial patterns and distributions of both micro-scales are quite similar; higher values are found in the vicinity of the upper impeller jet and the liquid free-surface. The Taylor micro-scales lie between $0.5 \mathrm{~mm}$ and $5 \mathrm{~mm}$ within the measurement plane with $\overline{\lambda_{r r}}=2.6 \mathrm{~mm}$ and $\overline{\lambda_{z z}}=2.4 \mathrm{~mm}$; this order of magnitude is coherent with the spatial resolution of the PIV technique $(\Delta x=0.6 \mathrm{~mm})$ which is thus small enough to capture the micro-scales $\lambda_{i i}$ and to accurately estimate the kinetic energy $k$.

The Taylor macro and micro length-scales are thus functions of location in the vessel; both increase towards the liquid freesurface with multiplying factors in the range $\times 1.5-\times 3$. These hydrodynamic length-scales thus reveal a significant stretching of eddies near the air-water interface, exactly where the mass transfer is happening. As the effect of the liquid free-surface on the length-scales is clearly shown (especially in their vertical extent), it seems reasonable to expect that large length-scale turbulent eddies could have a significant influence on surfacerenewal motions. Besides, the use of only one constant value for these macro- and micro-scales could lead to underestimations of other (turbulent) quantities, especially close to the top surface. For example, the use of single mean values for the 

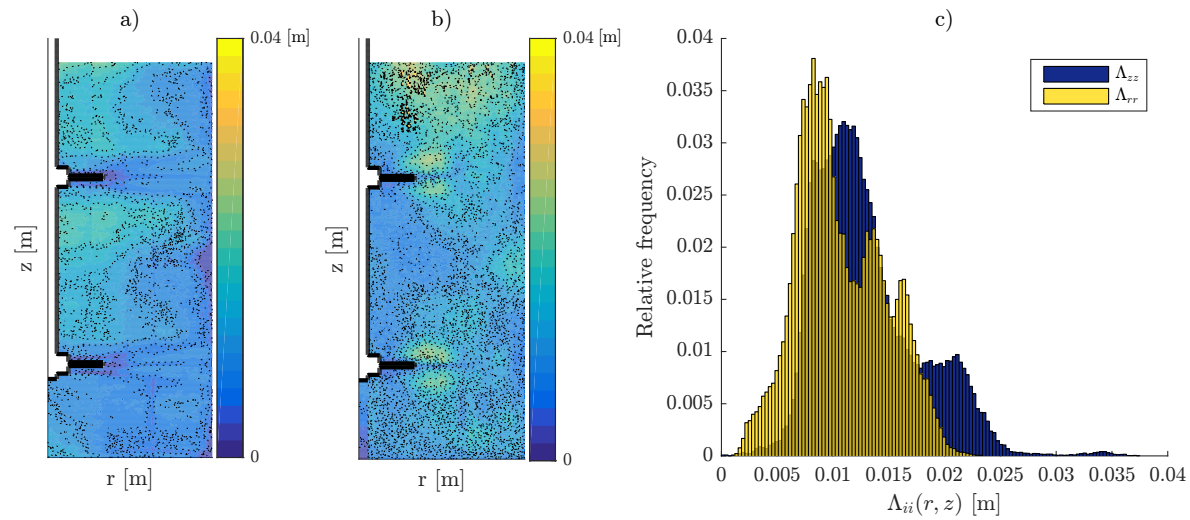

Figure 5: Longitudinal macro length-scales $\Lambda_{r r}$ (a) and $\Lambda_{z z}$ (b) maps and their distributions (c) on the measurement plane
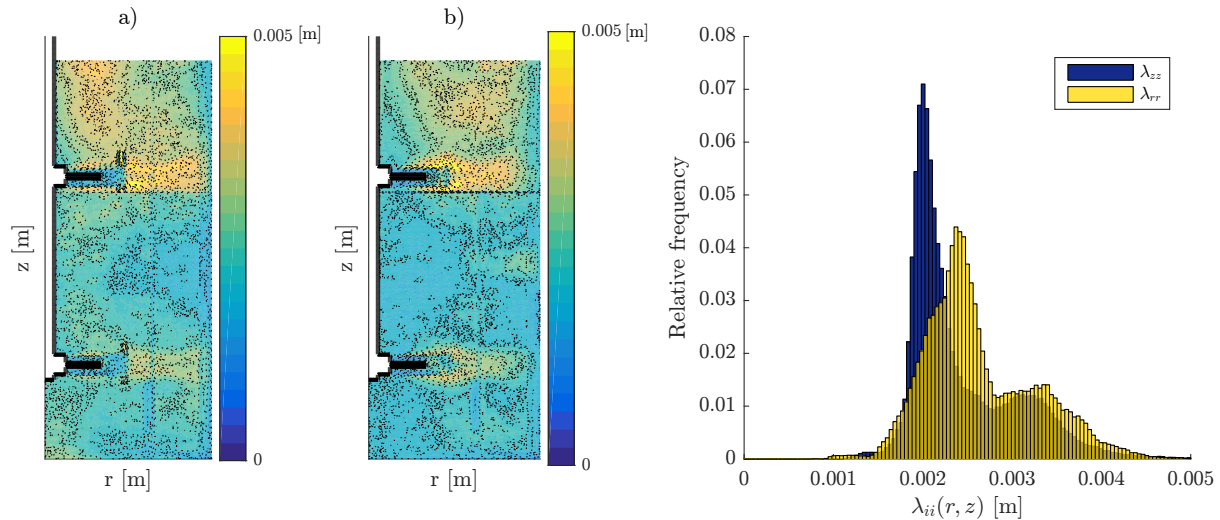

Figure 6: Longitudinal micro length-scales $\lambda_{r r}$ (a) and $\lambda_{z z}$ (b) maps and their distributions (c) on the measurement plane 
length-scales, especially the integral one $\Lambda$, is certainly not appropriate to depict the mechanism of mass transfer through the liquid free-surface.

These results lead to interrogations on the characteristics of turbulence and of the induced effects closed to the interface. Indeed, the bulk turbulence appears different from the one observed near the interface which also results in free-surface deformations. At $N=300 \mathrm{rpm}$, the interface is rather wavy and all stabilizing effects of the surface tension and gravity seem to have disappeared. Large-scale vertical flow motions induced by the baffles are then reduced in the vicinity of the free-surface, and their energy is transferred towards horizontal velocity fluctuations and surface distortions. Indeed, the presence of the gas-liquid interface emphasizes small scales for both velocity components. This subsurface turbulence seems to indicate that mass transfer and transport are controlled by a dual regime behavior with the contributions of both small and large eddies, whose relative importance could be quantified on the basis of data acquired at different Reynolds numbers.

\subsection{Mixing}

Qualitative analysis of the tracer dispersion dynamics is performed by observing instantaneous concentration maps and their corresponding concentration distributions as reported in Figure 7 .

At the beginning of the experiment, just at the time of injection, the concentration distribution consists of a high narrow peak around zero. In the first stage of the mixing process, high concentration stream is transported by the flow from the tracer injection point (at an angular position opposite to the measurement plane) toward the upper impeller region. The tracer plume hits the turbulent flow generated by the first rotating Rushton turbine, then is strongly mixed and pushed away from the measurement plane because of the action of the tangential velocity component. At first, the tracer dispersion mainly invades the upper part of the stirred-tank and is gradually transferred to the lower one toward a homogeneous distribution. While the tracer is passing through up and down, the distribution shape changes and then evolves into another peak corresponding to a uniform concentration in the whole tank. It is worth noting that, during the first phases of the experiment $(t=0.5-1 \mathrm{~s})$, the concentrations in the tracer plume are higher than $5 \mu \mathrm{g} / \mathrm{L}$ (histograms) and even than $10 \mu \mathrm{g} / \mathrm{L}$ (maps). These limits have been chosen to emphasize the tracer dispersion dynamics in the whole measurement plane. Significantly higher values than the average in the plane are due to image noise and occasional laser reflections on impellers and the liquid free-surface. This effect is noted during all the experiments.

Most batch mixing processes aims at making a spatially homogeneous product using the minimum of energy, and/or in the minimum of time. The degree of spatial mixing is generally judged through the variation of concentration of the inert tracer, which involves a proper sampling procedures and methods of quantifying the mixing state.

When dealing with PLIF measurements, it is worth summarizing all concentration inhomogeneities quantitatively. Two quantities are used in mixing literature to evaluate the intensity of segregation:

- the time needed to achieve a certain degree (95\%) of stability for the concentration field (time concept), i.e. the mixing time $t_{\mathrm{M}}$, based on the decay of the normalized concentration variance $\sigma_{\mathrm{M}, \mathrm{rms}}^{2}(t)$ (cf. Equation 2):

$$
\begin{gathered}
t_{\mathrm{M}}=t \text { for } \sigma_{\mathrm{M}, \mathrm{rms}}^{2}(t)=(0.05)^{2} \\
\text { with } \sigma_{\mathrm{M}, \mathrm{rms}}^{2}(t)=\frac{1}{N_{p i x}} \sum_{i=1}^{N_{p i x}}\left(\frac{C_{\mathrm{M}, i}(r, z, t)-C_{\mathrm{M}, i}\left(r, z, t_{0}\right)}{C_{\mathrm{M}, i}\left(r, z, t_{\infty}\right)-C_{\mathrm{M}, i}\left(r, z, t_{0}\right)}-1\right)^{2}
\end{gathered}
$$

- the time needed to achieve a certain degree (95\%) of concentration field uniformity (spatial concept), $t_{\mathrm{CoV}}$, based on the decay of the instantaneous concentration variance, namely the coefficient of variation $\operatorname{CoV}(t)$ (Hartmann et al. (2006), Kukuková et al. (2008), Busciglio et al. (2014), Busciglio et al. (2015)):

$$
\begin{gathered}
t_{C o V}=t \text { for } \operatorname{CoV}^{\prime 2}(t)=\left(\frac{\operatorname{CoV}(t)-\operatorname{CoV}\left(t_{\infty}\right)}{\operatorname{CoV} V_{\max }-\operatorname{CoV}\left(t_{\infty}\right)}\right)^{2}=(0.05)^{2} \\
\text { with } \operatorname{CoV}(t)=\sqrt{\frac{1}{N_{p i x}} \sum_{i=1}^{N_{p i x}}\left(\frac{C_{\mathrm{M}, i}(r, z, t)}{\overline{C_{\mathrm{M}}(t)}}-1\right)^{2}} \\
\text { where } \overline{C_{\mathrm{M}}(t)}=\frac{1}{N_{p i x}} \sum_{j=1}^{N_{p i x}} C_{\mathrm{M}, j}(r, z, t)
\end{gathered}
$$

where $\operatorname{CoV}\left(t_{\infty}\right)$ and $\operatorname{CoV}_{\max }$ are respectively the values of $\mathrm{CoV}$ when the injected tracer stream starts invading the measurement plane and when perfect homogenization is achieved.

These two "mixing times", $t_{\mathrm{M}}$ and $t_{\mathrm{CoV}}$, are similar in their definition. However, they are not based on the same approach. The first mixing time $t_{\mathrm{M}}$ is the time required for obtaining a stationary concentration field for which the time fluctuations of concentration become negligible, and $\sigma_{\mathrm{M}, \mathrm{rms}}^{2}(t)$ can be considered as the "distance" from this stationary field theoretically corresponding to a spatially uniform concentration field. On the other hand, the second mixing time $t_{\mathrm{CoV}}$ directly correspond to the time required for obtaining a uniform concentration field and $\operatorname{CoV}^{\prime}(t)$ can be considered as the "distance" from this uniform field.

Figure 8 presents the $t_{\mathrm{M}}$ dynamics measured by the PLIF technique for three injections and by the conductivity technique. The label "injection 1 " corresponds to the concentration maps and distributions of Figure 7. The global and local techniques give results within the same range as expected. They do not perfectly match due to their sampling strategy (PLIF: 2D vs. Conductivity: 1D) (Kukuková et al. (2008)). The associated sample number and size are quite different (PLIF: $3.5 \cdot 10^{6}$ pixels with $\Delta t=0.04 \mathrm{~s} v s$. Conductivity: 3 points of measurements with $\Delta t=0.5 \mathrm{~s}$ ). As regards the conductivity probe size used in this work, it corresponds to a quite important measurement surface and then it is assumed that the concentration is averaged over the probe volume estimated at $12 \mathrm{~mL}$. The curves from 


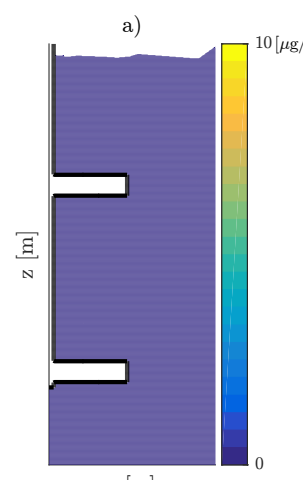

$\mathrm{r}[\mathrm{m}]$

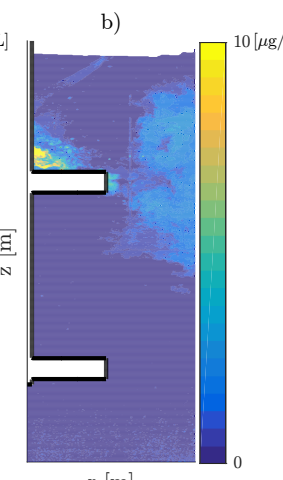

$\mathrm{r}[\mathrm{m}]$

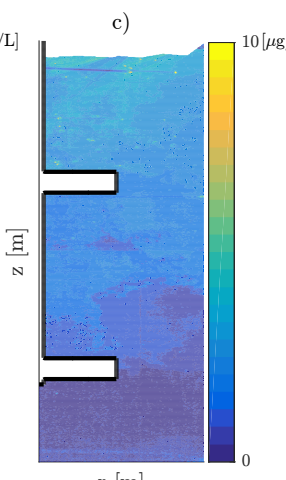

$\mathrm{r}[\mathrm{m}]$

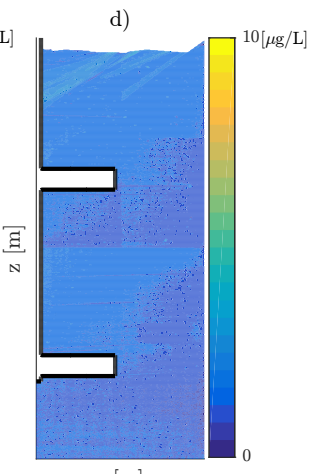

$\mathrm{r}[\mathrm{m}]$

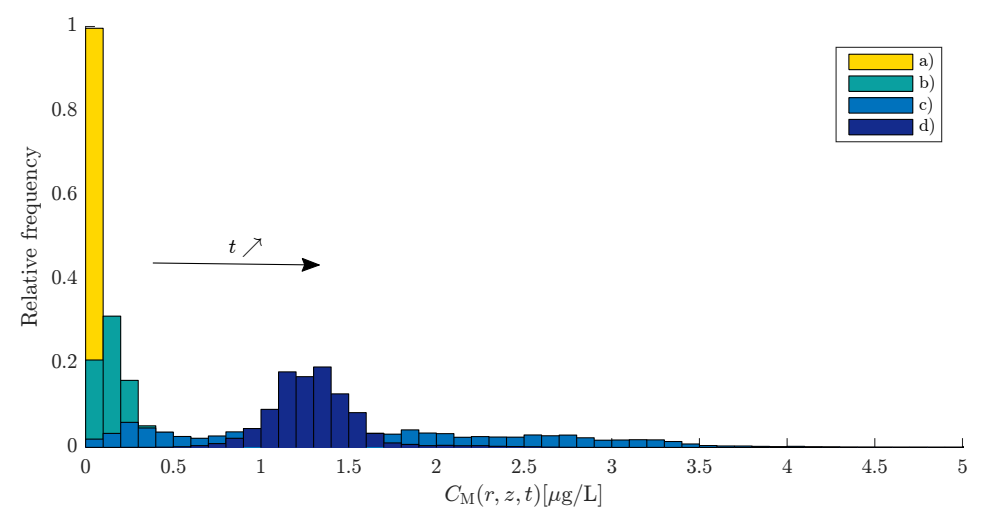

Figure 7: Sequence of instantaneous $C_{\mathrm{M}}(r, z, t)$ maps and distributions after injection of tracer solution at the free-surface at an angular position opposite to the measurement plane $(\Delta t=15 \mathrm{~s}, t=0 \mathrm{~s}(\mathrm{a}), 1 \mathrm{~s}(\mathrm{~b}), 3 \mathrm{~s}(\mathrm{c})$ and $15 \mathrm{~s}(\mathrm{~d}))$

PLIF data differ from each other mainly for two reasons. On one hand, due to pixel size and acquisition frequency, they are affected by turbulent concentration fluctuations. On the other hand, as already mentioned, image noise and laser reflections disturb the measurements.

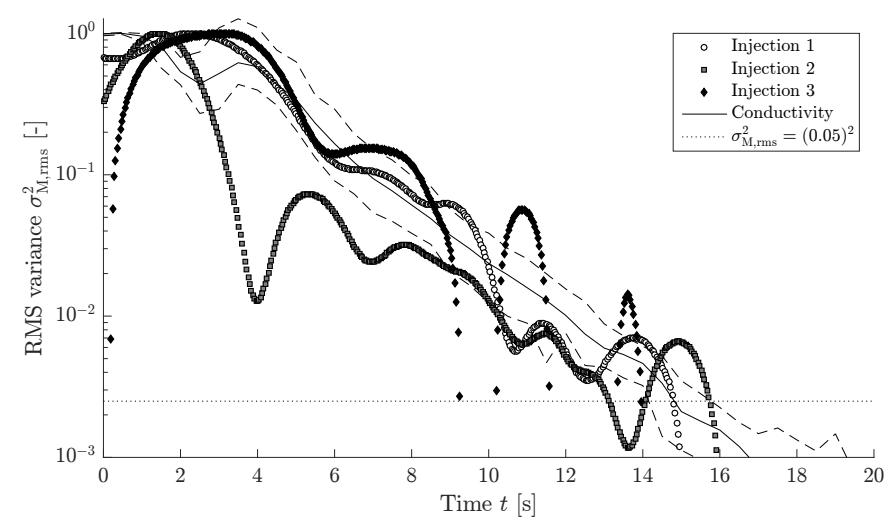

Figure 8: Instantaneous values of r.m.s. variance $\sigma_{\mathrm{M} \text {,rms }}^{2}$ (markers: PLIF data, dash-limited area: conductivity data, dotted line: $\left.\sigma_{\mathrm{M}, \mathrm{rms}}^{2}=(0.05)^{2}\right)$

Comparison of values obtained with global and local techniques is performed by observing the spatial distribution of local mixing times as reported in Figure 9. These results are obtained while looking at each pixel individually and averaging the ob- tained values for three subsequent injections:

$$
\begin{gathered}
\overline{t_{\mathrm{M}}}(r, z)=\frac{1}{3} \sum_{i=1}^{3} t_{\mathrm{M}, i}(r, z) \\
\text { where } t_{\mathrm{M}, i}(r, z)=t \text { for } \sigma_{\mathrm{M}, i}^{2}(r, z, t)=(0.05)^{2} \\
\text { with } \sigma_{\mathrm{M}, i}^{2}(r, z, t)=\left(\frac{C_{\mathrm{M}, i}(r, z, t)-C_{\mathrm{M}, i}\left(r, z, t_{0}\right)}{C_{\mathrm{M}, i}\left(r, z, t_{\infty}\right)-C_{\mathrm{M}, i}\left(r, z, t_{0}\right)}-1\right)^{2}
\end{gathered}
$$

This mixing time field $\overline{t_{\mathrm{M}}}(r, z)$ indicates that mixing dynamics is not homogeneous in the whole tank, but the global mixing time value of $14 \mathrm{~s}$ calculated from the conductivity measurements turns out to be a correct estimate. The positions of the conductivity probes turn out to be well-representative of the system mixing behavior. The region between the two impellers and at the bottom are identified by PLIF as, respectively, the $\operatorname{most}\left(\overline{t_{\mathrm{M}}}(r, z) \in[5 \mapsto 10] \mathrm{s}\right)$ and the least efficient mixing zone $\left(\overline{t_{\mathrm{M}}}(r, z) \gg 15 \mathrm{~s}\right)$.

The $C o V^{\prime}$ dynamics is shown in Figure 10. Three time sequences have been identified and are separated through the dashed vertical lines. In the first stage of the process, the $\mathrm{CoV}^{\prime}$ is steeply increasing from its initial value to its maximum one as the tracer, coming from its diametrically opposed injection point, invades the illuminated area more and more. In the second stage, the high concentration stream is broken up by the upper impeller and mixed into smaller structures with lower concentration mainly into the upper part of the tank. At the 

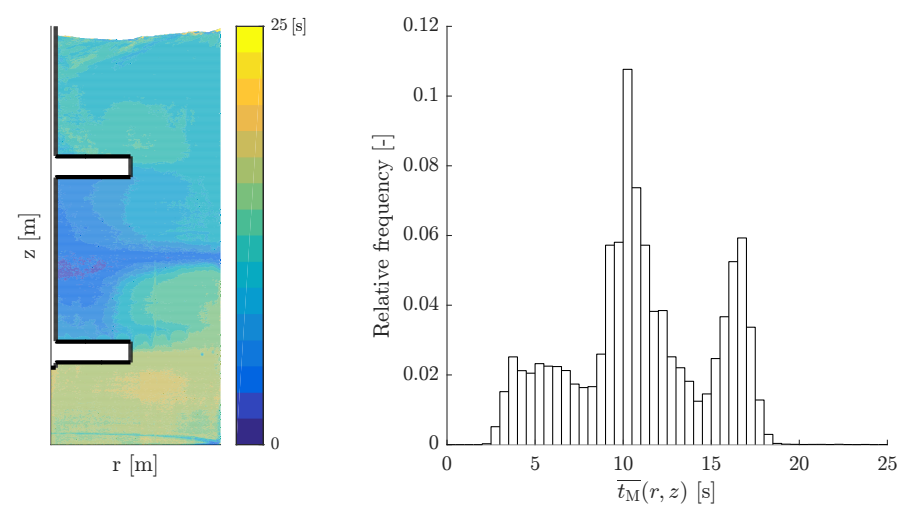

Figure 9: Local mixing time $\overline{t_{\mathrm{M}}}(r, z)$ map and distribution averaged over three injections

final stage, the graph reveals a decay of the $C o V^{\prime}$. The tracer is exchanged between the upper and lower zones of the tank, mainly including turbulent diffusion. This leads to net transport of dye between the two regions, resulting in homogenization. When homogenization is nearly complete, most pixels have the same concentration values. The $C o V^{\prime}$ tends then to values close to zero. However, some oscillations are observed in the semilog plot reported in Figure 10 because of image noise and laser reflections as already mentioned.

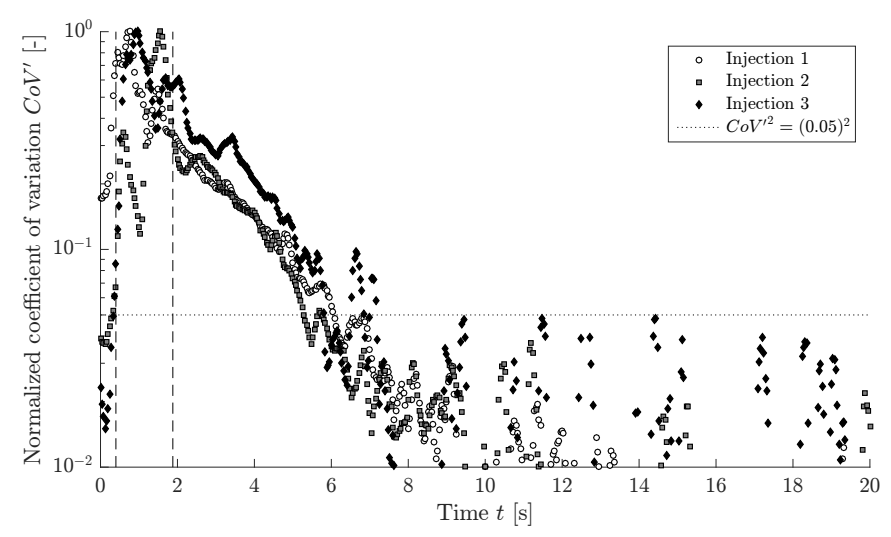

Figure 10: Instantaneous values of normalized coefficient of variation $\mathrm{CoV}^{\prime}$ (markers: PLIF data, dashed line: time intervals, dotted line: $\left.\mathrm{CoV}^{2}=(0.05)^{2}\right)$

The two "mixing times" used in the present work to characterize the mixing process do not have the same value: around $15 \mathrm{~s}$ for the mixing time $t_{\mathrm{M}}$ and $7 \mathrm{~s}$ for the $\mathrm{CoV}$ time $t_{\mathrm{CoV}}$ as shown in Figures 8 and 10. The characterization of mixing efficiency using one single parameter is hardly possible due to the complexity of the phenomenon, as already pointed out in literature (Kukukova et al. (2011), Alberini et al. (2014), Montante et al. (2016)). Different quantification procedures, primarily based on the time or spatial evolution of the concentration field for example, logically lead to different parameter values even if these parameters have the same name. However, these two time parameters are complementary and worthy of interest for the evaluation of mixedness and consequently the discrimination between agitation systems.
The intensity of segregation is often the sole criterion for characterizing mixing efficiency. However, this is just one dimension of the problem as it only quantifies the extent of concentration variation as stated in the general mixing concepts introduced by Danckwerts (1952) and lately developed by Kukukova et al. (2009). An additional dimension is generally the scale of segregation to describe the size of unmixed regions. Its measure adopted by Danckwerts (1952) is analogous to the integral scale used in Section 3.2:

$$
\begin{aligned}
R_{\mathrm{M}_{r}}(d r, t)= & \frac{\overline{\left(C_{\mathrm{M}}(r, z, t)-\overline{C_{\mathrm{M}}}(t)\right) \cdot\left(C_{\mathrm{M}}(r+d r, z, t)-\overline{C_{\mathrm{M}}}(t)\right)}}{\sigma_{\mathrm{M}}^{2}(t)} \\
& \Rightarrow \Lambda_{\mathrm{M} r}(t)=\int_{0}^{\infty} R_{r} \cdot(d r, t) d r \\
R_{\mathrm{M} z}(d z, t)= & \frac{\overline{\left(C_{\mathrm{M}}(r, z, t)-\overline{C_{\mathrm{M}}}(t)\right) \cdot\left(C_{\mathrm{M}}(r, z+d z, t)-\overline{C_{\mathrm{M}}}(t)\right)}}{\sigma_{\mathrm{M}}^{2}(t)} \\
& \Rightarrow \Lambda_{\mathrm{M} z}(t)=\int_{0}^{\infty} R_{z}(d z, t) \cdot d z
\end{aligned}
$$

where $\overline{C_{\mathrm{M}}}(t)$ and $\sigma_{\mathrm{M}}^{2}(t)$ are the mean and the variance of the data of the whole measurement plane of at a given moment $t$.

These length scales of segregation represent the greatest length at which unmixed material can be detected (Rielly et al., 1994). The horizontal and vertical length scales calculated after injection of tracer solution at the free-surface at an angular position opposite to the measurement plane are shown in Figure 11.

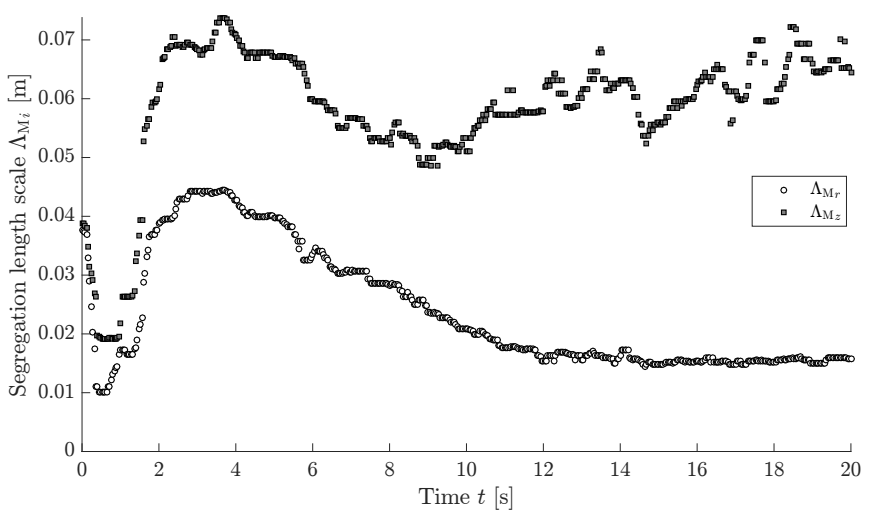

Figure 11: Instantaneous values of segregation lengthscales $\Lambda_{\mathrm{M} r}$ and $\Lambda_{\mathrm{M} z}$ after injection of tracer solution at the free-surface at an angular position opposite to the measurement plane

Similarly, both scales initially increase as the injected fluid begins to fill the vessel, reach a peak, and decrease as the tracer disperses in the whole vessel. The vertical length scale is larger than the horizontal one due to the high aspect ratio of the system. The length scales at the end of the experiment, in particular the vertical one, are quite disturbed by the noise generated by laser reflections on the free-surface and then do not tend to some small values as it could be expected. Therefore, from Figure 11, it can be inferred that these mean length scales correctly track the progress over time to a homogenized concentration field, but contains very little information about the complex mixing 
structures in the measurement plane, which necessarily contain a wide distribution of length scales.

The horizontal length scale $\Lambda_{\mathrm{M} r}$ falls and stays within $\pm 5 \%$ of its final value after $c a .14 \mathrm{~s}$, which is equal to the mixing time $t_{\mathrm{M}}$ and is twice the the $\mathrm{CoV}$ time $t_{\mathrm{CoV}}$. The time needed to achieve stability for the concentration field (r.m.s. variance $\sigma_{\mathrm{M}, \mathrm{rms}}^{2}$ ) is indeed influenced by the time evolution of the average size of unmixed clumps within the mixture (scale of segregation $\Lambda_{M}$ ) and of the concentration differences between these clumps of fluid (intensity of segregation $\mathrm{CoV}$ ). In the present case, the reduction in length scale of segregation appears to be rate-determining of the mixing process.

These considerations on mixing performance seem to indicate that a step variation of oxygen concentration in the headspace leads to the formation, in the dissolved oxygen concentration field, of clumps of uniform but differing compositions. The composition differences between zones are however weak given the slowness of the mass transfer process estimated from global measurements.

\subsection{Mass transfer}

Comparison of values obtained with global and local techniques is first performed by observing the mass transfer coefficient map and its corresponding distribution reported in Figure 12. These results are obtained by looking at each pixel individually and then averaging three desorption experiment results. The parameter value estimated for each pixel of the measurement plane is the $K_{\mathrm{L}} a$ value obtained using Equation (3). For pixels not belonging to the free surface, no interfacial gas-liquid area may be defined and the estimated $K_{\mathrm{L}} a$ value has thus no real physical meaning. It just refers to the time dynamics of the local concentration evolution. Despite this, it has been chosen to continue referring to it as $K_{\mathrm{L}} a$ for comparison purposes, to avoid the introduction of an additional parameter. This mass transfer coefficient field $\overline{K_{\mathrm{L}} a}(r, z)$ indicates that mass transfer appears generally almost homogeneous in the whole tank and that the dynamics of the process is quite slow. Higher values are observed in the upper part of the tank, which may be linked to the spatial distributions of the turbulent length-scales. The significant stretching of eddies most likely induce surface renewal motions, precisely just where the driving force of gasliquid transfer (dissolved oxygen gradient) is the largest.

Another analysis may be done by considering the whole measurement plane and combining $N_{\text {pix }}$ pixel responses. The results are given in Table 1 with the mean of dynamic method measurements performed at the same time. The global and local techniques give results within the same order of magnitude. The global mass transfer coefficient value of $4.4 \cdot 10^{-4} \mathrm{~s}^{-1}$ calculated from the dynamic method measurements turns out to be a slightly higher estimation than the present PLIF results. They do not exactly match, among other reasons, due to their sampling strategy as previously explained for the mixing results (number and size of measurement points). Concerning the dissolved oxygen probe used in this work, it is a quite sensitive microsensor for which only the tip can be immersed thus limiting its location to the surroundings of the free surface where mass transfer mainly takes place.
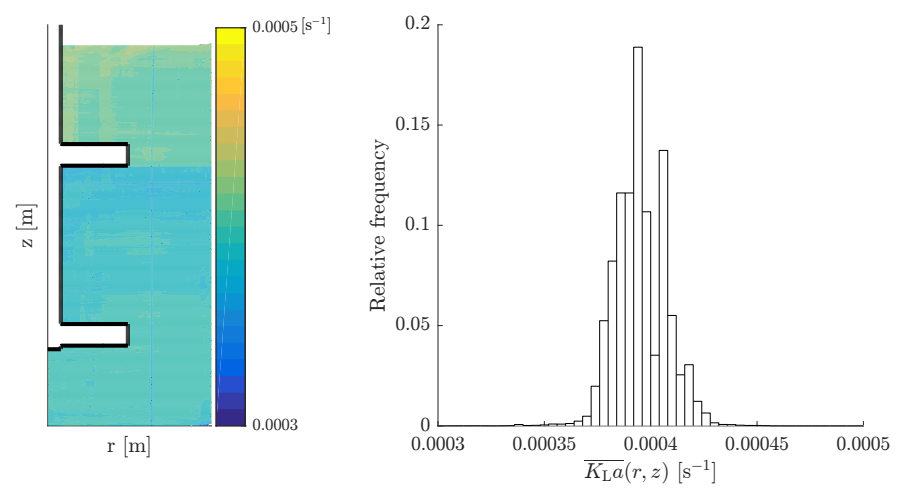

Figure 12: Local mass transfer coefficient $\overline{K_{\mathrm{L}} a}(r, z)$ distribution averaged on three desorption experiments

Table 1: Comparison of global mass transfer coefficient values measured by the PLIF and the dynamic methods

\begin{tabular}{|l|c|}
\hline \multicolumn{2}{|l|}{ Global mass transfer coefficient $K_{\mathrm{L}} a\left[\mathrm{~s}^{-1}\right]$} \\
\hline PLIF 1 & $3.8 \cdot 10^{-4}$ \\
PLIF 2 & $4.0 \cdot 10^{-4}$ \\
PLIF 3 & $4.1 \cdot 10^{-4}$ \\
\hline Probe & $4.4 \cdot 10^{-4} \pm 3 \%$ \\
\hline
\end{tabular}

Qualitative analysis of the dissolved oxygen dynamics is performed by observing instantaneous concentration maps and their corresponding concentration distributions as reported in Figure 13. At the beginning of the experiments, the bulk had been fully oxygenated and the mean equilibrium dissolved oxygen concentration with pure $\mathrm{O}_{2}$ is $c a .45 \mathrm{mg} / \mathrm{L}$. The concentration field values are, as expected, decreasing with time. Each image is dominated by mainly one color-scale corresponding to the mean oxygen concentration in the bulk, which is constantly mixed by the liquid flow motions generated by the impeller rotation. On several concentration maps of Figure 13, strikes appear due to fleeting laser light distortions on the liquid freesurface (experimental noise) and cause then erroneous concentration values. At each time, the predominant color-scale is also indicated by the highest peak of the corresponding histogram on Figure 13. As the desorption process goes on, the exchange potential $\left(C_{\mathrm{O}_{2}}^{*}-C_{\mathrm{O}_{2}}(t)\right)$ (Eq.(3)) becomes smaller before reaching the equilibrium with air, $C_{\mathrm{O}_{2}}^{*} \simeq 9 \mathrm{mg} / \mathrm{L}$. At any moment, there is then a distribution of dissolved oxygen concentration values. This measured non-uniformity is not only due to experimental noise. Indeed, while they are logically moving towards smaller values when $t$ increases, the histograms also become narrower. The variance decreases by a factor of 100: its value is around $3(\mathrm{mg} / \mathrm{L})^{2}$ at the beginning of the desorption and around 0.03 $(\mathrm{mg} / \mathrm{L})^{2}$ at the end of the PLIF measurements (which is about the variance estimated from calibration data). Therefore, it corresponds to real $\mathrm{O}_{2}$ gradients that decrease as the dissolved oxygen concentration tends towards its equilibrium value. Indeed, at that time, the mass transfer process is so slow that it can no longer induce gradients which the mixing process does not have time to cancel. 

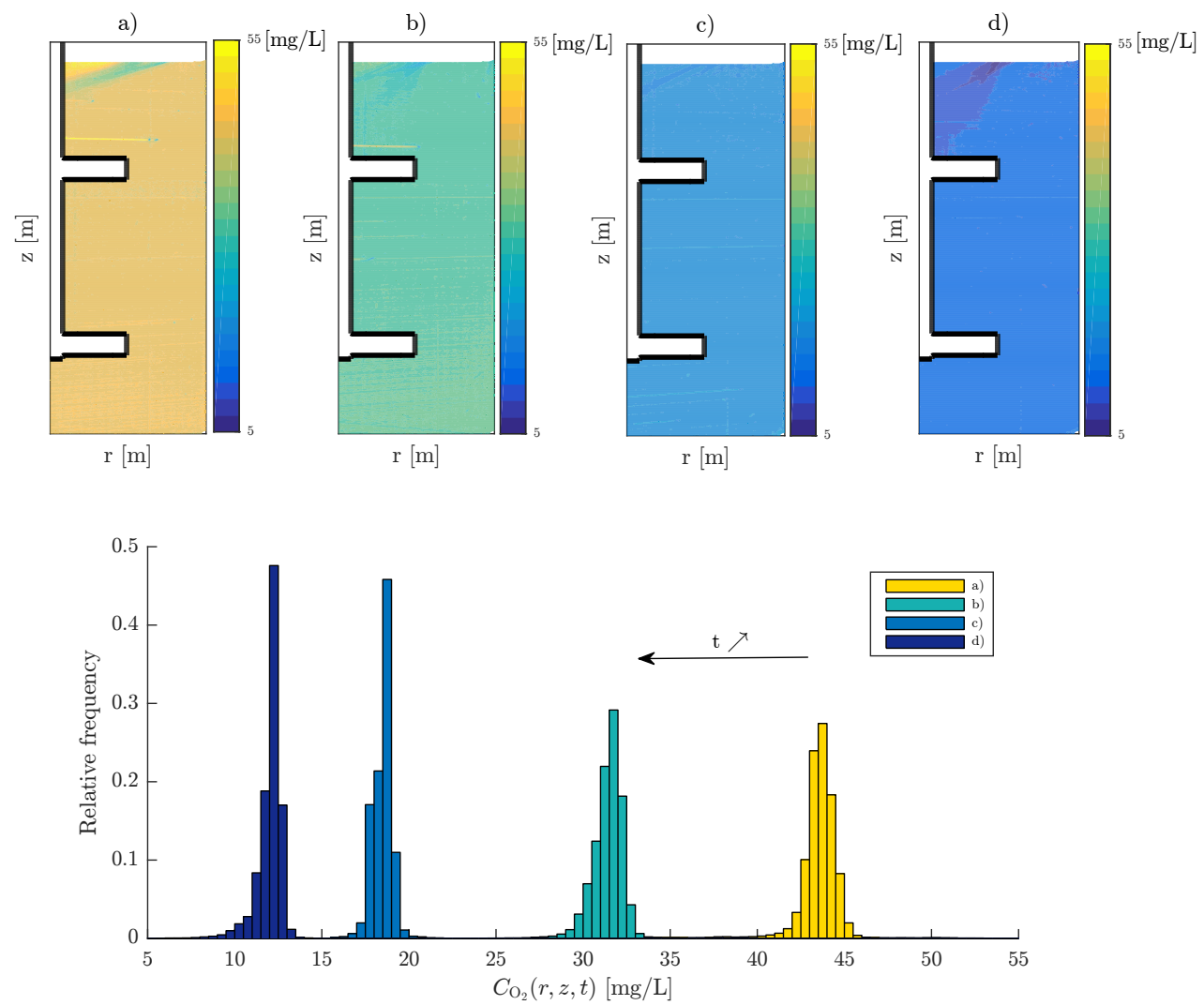

Figure 13: Sequence of instantaneous $C_{\mathrm{O}_{2}}(r, z, t)$ maps and distributions over a long period ( $\Delta t=120 \mathrm{~min}, t=0 \mathrm{~min}(\mathrm{a}), 20 \mathrm{~min}$ (b), $60 \mathrm{~min}$ (c) and $120 \mathrm{~min}$ (d)) after step variation of oxygen concentration (from pure oxygen to air) in the headspace

It is also interesting to observe the gas exchange process during a shorter period of time (15 s instead of $120 \mathrm{~min})$ as depicted in Figure 14.a. measured at different periods of a desorption experiment. The first one $\left(t_{1}+\Delta t=33 \mapsto 48 \mathrm{~s}\right)$, on the right, corresponds to the very beginning of the experiment $( \pm 0 \%$ of desorption). The second one $\left(t_{2}+\Delta t=1350 \mapsto 1365 \mathrm{~s}\right)$, at the middle, corresponds to $45 \%$ of desorption, while the third one $\left(t_{3}+\Delta t=2440 \mapsto 2455 \mathrm{~s}\right)$ on the left, corresponds to $75 \%$ of desorption. Each group is composed of six profiles measured over a period of $15 \mathrm{~s}$ with a time step of $3 \mathrm{~s}$. The period of $15 \mathrm{~s}$ was chosen in agreement with the mixing time value. The corresponding instantaneous $C_{\mathrm{O}_{2}}(r, z, t)$ maps are not presented because there are no perceptible changes between them. However, there are significant shifts in the concentration distributions while $\Delta t \simeq t_{\mathrm{M}} \ll 1 / K_{\mathrm{L}} a$. These shifts do not come from experimental noise. Indeed, the laser light distortions lead to significantly smaller values than the range of concentrations displayed on the histograms of Figure 14.a. The histograms shape tends to follow a cyclic pattern. The peaks slightly decrease and increase all the while moving to the left. During the desorption process, flow motions continuously renew fluid elements near the interface which cause spatial heterogeneities even though for a length of time $\Delta t \simeq t_{\mathrm{M}}$ the concentration field seems stationary.

Figure 14.b. presents three groups of vertical profiles of the dissolved oxygen concentration at the three above-mentioned periods of the desorption experiment. It is worth noting that, during these three sequences of $15 \mathrm{~s}$, there were no laser distortions near the selected axial profile $(r=0.0917 \mathrm{~m})$. The concentration profiles are thus not affected by noise and their fluctuations really correspond to dissolved oxygen concentration fluctuations.

One may observe that $(i)$ the concentration profiles are not constant along depth and (ii) their time evolution within a given group, relative to short time scales, is not monotonous at all.

The vertical gradients extend over the whole height of the tank. They are not limited within a small boundary layer just below the liquid free-surface. It means that, despite a high mixing efficiency (short mixing time), the dissolved oxygen concentration is not perfectly homogeneous in the bulk. So, even if mixing and mass transfer dynamics are very different, as assessed by the huge difference between their global characteristic times $\left(t_{\mathrm{M}}=14 \mathrm{~s} \ll 1 / K_{\mathrm{L}} a=2500 \mathrm{~s}\right.$ ), the mass transfer process inside a stirred-tank reactor aerated through the liquid free-surface can thus not be modeled by simply coupling a perfectly-mixed bulk and a thin concentration boundary layer.

Within a given group of vertical $C_{\mathrm{O}_{2}}$ profiles, fluctuations with time for pixels situated between the free-surface $(z=0.44 \mathrm{~m})$ and the upper impeller $(z=0.38 \mathrm{~m})$, may be important despite the short time step between them and seem completely random. Figure 14.c shows the standard deviation profiles relative to the three groups of concentration profiles of Figure 14.b. For each group, the standard deviation $\sigma_{\mathrm{O}_{2}}$ is higher near the interface. The observation of these standard deviation profiles 

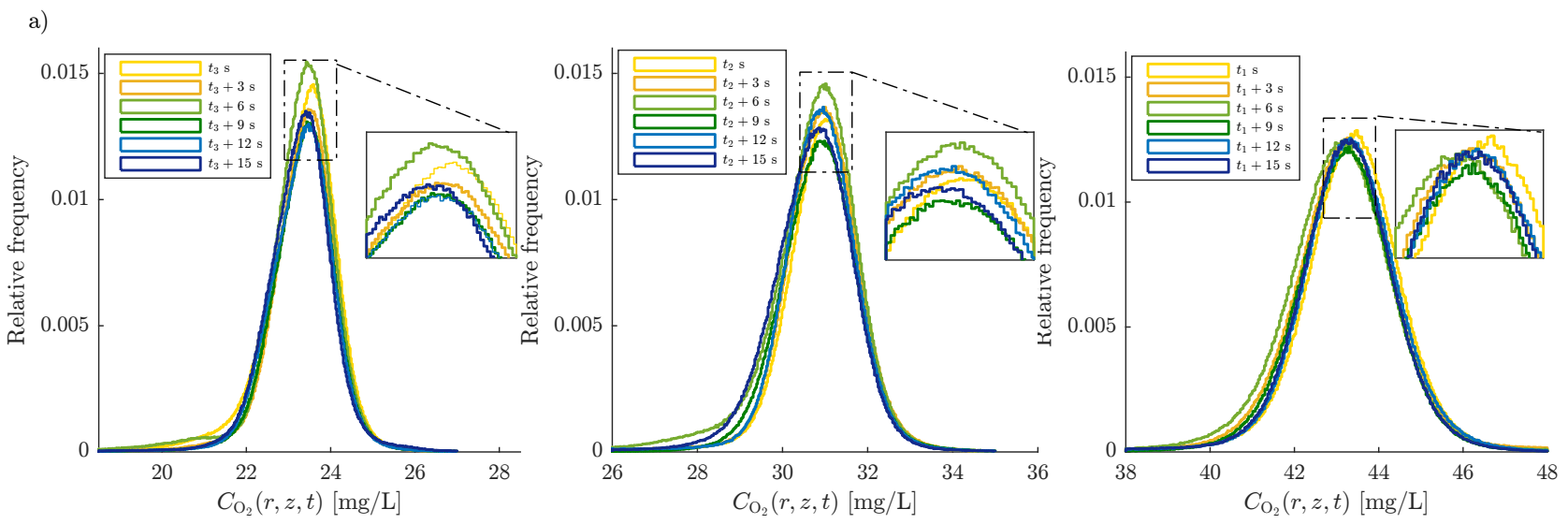

b)

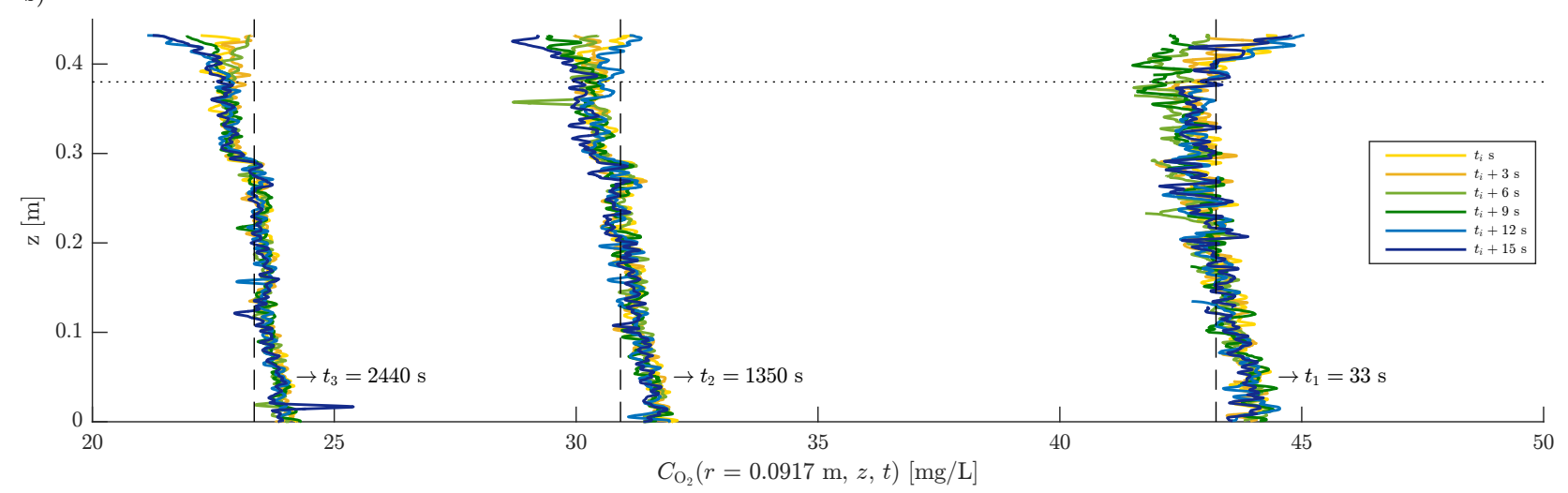

c)
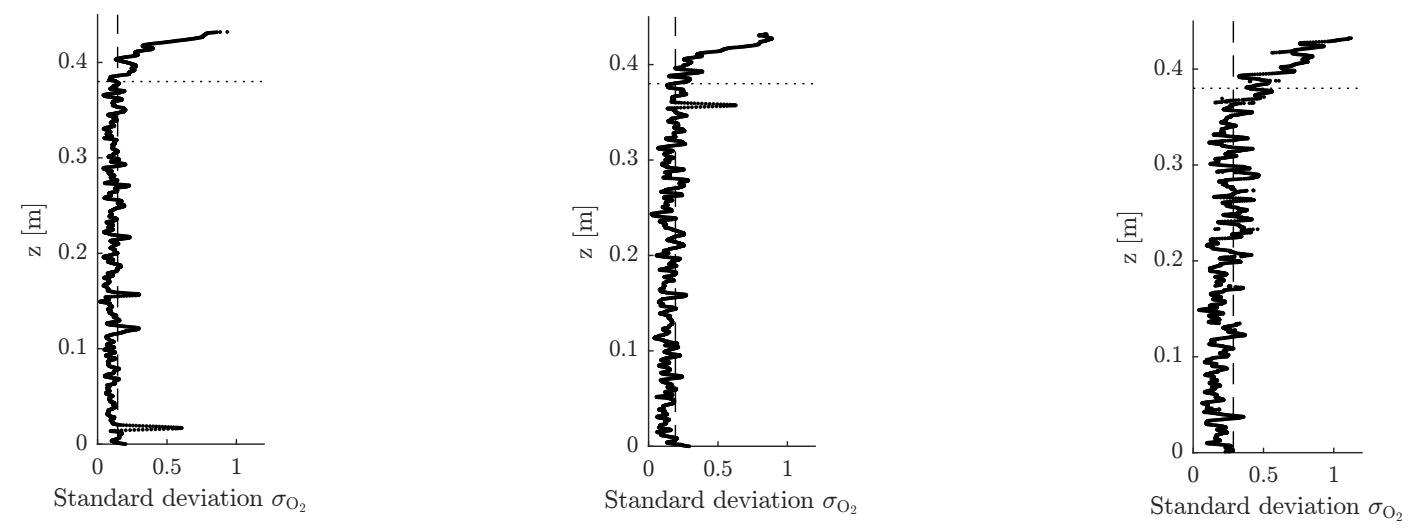

Figure 14: Time evolution over a period of $\Delta t=15 \mathrm{~s}$ by a time step of $3 \mathrm{~s}$ at three different moments of the desorption process, $t_{1}=33 \mathrm{~s}, t_{2}=1350 \mathrm{~s}$ and $t_{3}=2440 \mathrm{~s}$, of dissolved oxygen concentration distributions (a), axial profiles - $r=0.0917 \mathrm{~m}(\mathrm{~b})$ and their corresponding standard deviation profiles (c)(continuous lines - PLIF data, dashed lines - mean concentration in the whole measurement plane for $t_{i}+\Delta t$ ), dotted line - constant $z=0.38 \mathrm{~m}$ 
determines the transition between the "interfacial region" and the "bulk region" with more accuracy. The limit may be detected at a height equal to $0.38 \mathrm{~m}$, i.e. at a distance of 0.06 $\mathrm{m}$ from the free-surface. This thickness of $c a .0 .06 \mathrm{~m}$ of this "interfacial region" suggests that large-scale eddy motions with size ranges between the micro- and macro-scales (cf. section 3.2: $\lambda \in\left[5 \cdot 10^{-4}, 5 \cdot 10^{-3}\right] \mathrm{m}$ and $\Lambda \in\left[1.5 \cdot 10^{-3}, 3.5 \cdot 10^{-2}\right]$ $\mathrm{m})$ are interfering in the surface renewal and thus in the gasliquid mass transfer. The spatial distributions of the turbulent length-scales $\left(\Lambda_{z z}, \lambda_{z z}\right.$ and $\left.\lambda_{r r}\right)$ have revealed larger eddies in the vicinity of the liquid free-surface. The extent of this zone of higher values can be estimated by image segmentation processing applied on length-scales maps. Its boundaries may be detected at a maximal height equal to $0.37 \mathrm{~m}$ for $\Lambda_{z z}, 0.36 \mathrm{~m}$ for $\lambda_{z z}$ and $0.35 \mathrm{~m}$ for $\lambda_{r r}$. These values are closed to the one of $0.38 \mathrm{~m}$ estimated from the PLIF results. Additional experiments with different Rushton turbine combinations (clearance, submergence and spacing) would be necessary to confirm that the area of the interfacial region can be estimated from the analysis of the length-scales maps.

The major concentration fluctuations in a region between the free-surface and the upper impeller may be explained by the presence of up-welling and down-welling large-scale motions at the vicinity of the surface which results in complex displacements of fluid elements. The largest concentration fluctuations could be associated to the action of up-welling motions, and the small ones to down-welling motions. Up-welling structures result from the ejection of fluid blobs from the bottom part of the tank which carry bulk fluid towards the surface. The vanishing of the vertical velocity at the surface forces these up-draughts to spread out and roll up, displacing a large amount of near-surface fluid. The collision of two neighboring up-draughts results in the creation of down-welling structures which carry fluid into the bulk. During the desorption process, up-welling and downwelling motions contribute to high and low concentration respectively. These large-scale flow structures rising from the underlying turbulence generated by the impellers on the nearsurface dynamics are depicted in Figure 15.

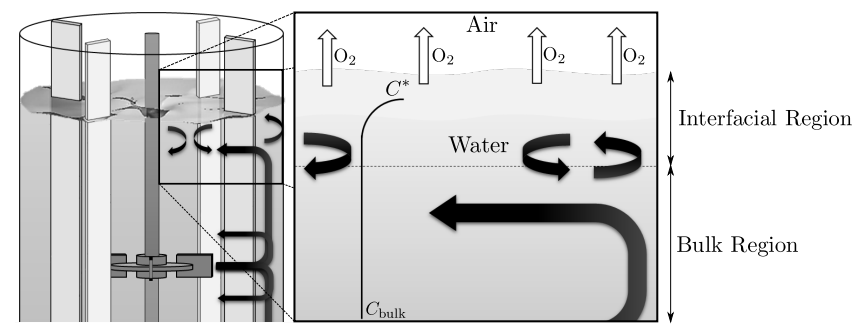

Figure 15: Schematic illustration depicting the gas-transfer through the liquid free-surface inside a stirred-tank bioreactor

Even if global measurements show that global characteristic times for mixing and mass transfer differ by two orders of magnitude, the dissolved gas concentration is not as perfectly uniform as one might expect. Flow dynamics and spatial heterogeneities, which are defined by geometric parameters and operating conditions, are ruling the underlying mechanisms of the mass transfer process through the liquid free-surface: the homogenization of the dissolved gas concentration (mixing efficiency) and the renewal of the liquid at the free-surface (circulation patterns).

The imposed conditions in the headspace (continuous supply of air) bring low-concentration fluid to the near surface region, whereas the initial conditions in the bulk fluid (saturation with pure oxygen) bring high concentration fluid to this region. All these flow structures (large eddies) lead to highly fluctuating concentration profiles near the air-water interface. At higher depths, concentration field is homogenized by turbulent stirring and is less fluctuating. The motions of eddy structures approaching the interface from below are thus an integral part of the transport mechanism in the present agitation system.

There is of course a broad and continuous spectrum of sizes for concentration structures. The large and small-scale structures discussed here are a simplified view of a much more complex reality whose complete description is not possible on the basis of available results within the frame of the present research.

\section{Conclusion}

The aim of this work was to globally and locally characterize a baffled 20L-vessel agitated by two Rushton turbines and aerated through the liquid free-surface, in order to investigate the effects of hydrodynamics and mixing on mass transfer. Global parameters $\left(P / V, t_{\mathrm{M}}, K_{\mathrm{L}} a\right)$ have been determined by means of accessible and simple experimental tools. Local quantities $\left(U_{r}(r, z, t), U_{z}(r, z, t), C_{\mathrm{M}}(r, z, t)\right.$ and $\left.C_{\mathrm{O}_{2}}(r, z, t)\right)$ have been measured in a vertical plane while using more advanced techniques, to wit PIV and PLIF.

First, the macroscopic quantities have been analyzed to choose the most relevant agitation condition in order to get detailed information on the system performance in terms of hydrodynamics, mixing and mass transfer. The selected stirring speed of $300 \mathrm{rpm}$ corresponds to a fully turbulent flow associated to a short mixing time, a moderate power consumption, a satisfactory mass transfer coefficient and, in particular, with no air entrainment from the liquid free-surface.

Then, the detailed data relative to a large volume fraction of the stirred tank obtained by PIV and PLIF give further insights into flow, mixing and mass transfer dynamics. The accuracy and reliability of the system calibration for both PLIF procedures $\left(C_{\mathrm{M}}(r, z, t)\right.$ and $\left.\left.C_{\mathrm{O}_{2}}(r, z, t)\right)\right)$ have been demonstrated for the whole considered concentration ranges $(0 \mapsto 80 \mu \mathrm{g} / \mathrm{L}$ and 0 $\mapsto 100 \% \mathrm{O}_{2}$ ) in the entire axial measurement plane.

In order to better understand the influence of hydrodynamics on mass transfer through the liquid free-surface, the Taylor macro and micro length-scales, $\Lambda$ and $\lambda$, have been estimated from the instantaneous velocity fields measured by PIV. These two quantities are functions of location in the vessel. They reveal a significant stretching of eddies near the air-water interface, exactly where the mass transfer is happening.

In order to assess the homogenization process within the vessel, the $C_{\mathrm{M}}(r, z, t)$ fields obtained by PLIF have been qualitatively introduced and quantitatively presented by means of averages, either in time or in space, and other statistical treatments. The mixing time field $\overline{t_{\mathrm{M}}}(r, z)$ indicates that mixing dynamics is not 
homogeneous in the whole tank and is correctly estimated by the global mixing time calculated from the conductivity measurements as both methods are based on the same approach (concentration field stability). Indeed, the characterization of homogenization process using one single parameter is hardly possible due to the complexity of the phenomenon. Different quantification procedures, primarily based on the time $\left(t_{\mathrm{M}}\right)$ or spatial evolution $\left(t_{\mathrm{CoV}}\right)$ of the concentration field lead to different parameter values. A proper evaluation of mixing performance implies consideration of both aspects.

The mass transfer analysis is carried out from the $C_{\mathrm{O}_{2}}(r, z, t)$ fields obtained from PLIF and is refined thanks to hydrodynamics and mixing outcomes. The mass transfer coefficient field $K_{\mathrm{L}} a(r, z)$ indicates that mass transfer appears generally almost homogeneous in the whole tank and that the dynamics of the process is quite slow. Higher values are observed in the upper part of the tank, which may be linked to the spatial distributions of the turbulent length-scales. The global mass transfer coefficient value estimated using a dissolved $\mathrm{O}_{2}$ probe located just below the free-surface is indeed slightly higher that one calculated from the PLIF results. The dissolved oxygen concentration profiles are not constant along depth and their time evolution is not monotonous at all.

Therefore, local concentration measurements show that the actual mechanism controlling the gas-liquid transfer process inside a stirred-tank reactor aerated through the liquid freesurface cannot be described by an over-simplified coupling of a perfectly-mixed bulk and a thin concentration boundary layer, as it might be assumed from the huge difference between the two characteristic global times of mixing and mass transfer dynamics $\left(t_{\mathrm{M}}=14 \mathrm{~s} \gg 1 / K_{\mathrm{L}} a=2500 \mathrm{~s}\right)$. Large-scale eddy motions are interfering in the surface renewal and in the interfacial mass transfer.

On the basis of the present outcomes, a phenomenological model comes out. The tank is divided in two zones: the "bulk region" where $C_{\mathrm{O}_{2}}(r, z, t)$ is homogenized by turbulent stirring and is less fluctuating, and the "interfacial region" where upwelling and down-welling large-scale motions interact to high and low concentration respectively, during the desorption process.

The coupled analysis of local velocity and concentration fields measured by PIV and PLIF has thus allowed a first step towards a more detailed analysis of the different phenomena. The present work has proven that a study only based on global parameters can be restrictive and unsatisfactory to describe spatial and time scales of the different phenomena.

Future work will include simultaneous measurements of velocity and concentration fields within a limited flow region under the liquid free-surface while increasing spatial resolution and paying attention to noise due to laser reflections. Conditional statistics of the velocity-concentration correlations (Janzen et al. (2010), Variano and Cowen (2013)) will be considered to further discuss the relative contribution of different spatial scales to the concentration and velocity gradients variations.

\section{Acknowledgements}

AdL is supported by a PDR FNRS PhD grant (PDR $\mathrm{n}^{\mathrm{o}}$ T.0250.13) from the National Funds for Scientific Research of Belgium (FRS-FNRS). AdL acknowledges Prof. V. Terrapon for the useful discussion on turbulent length-scales. All the authors gratefully thank T. Salmon and F. Fyon for their technical assistance.

\section{Appendix A. PLIF calibration}

\section{Appendix A.1. Mixing}

Before starting the experiments to measure the inert tracer instantaneous concentration field $C_{\mathrm{M}}(r, z, t)$, the range of linear response between the grayscale value and the tracer concentration (Eq.(6)) for each pixel has been determined by a calibration procedure, for which the tank was filled with Rh6G solutions of known concentrations. Subsequently, three runs $(1 \rightarrow 3)$ have been carried out in order to check the accuracy and reliability of the system calibration over the whole concentration range. For each run, the fluorescence intensity is recorded as a function of tracer concentrations for each pixel. As an example, the results plotted in Figure A.1 are relative to two measurement pixels, $\mathrm{P} 1$ and P2, respectively located in the upper and lower parts of the tank. These two zones do not receive the same amount of laser light due to technical specificities of the experimental system and devices, which leads to different fluorescence intensity values for the same tracer concentration. The three sets of points show the satisfactory reproducibility of the PLIF technique, which thus may be used to assess the mixing dynamics inside the stirred tank. The linearity has been observed up to

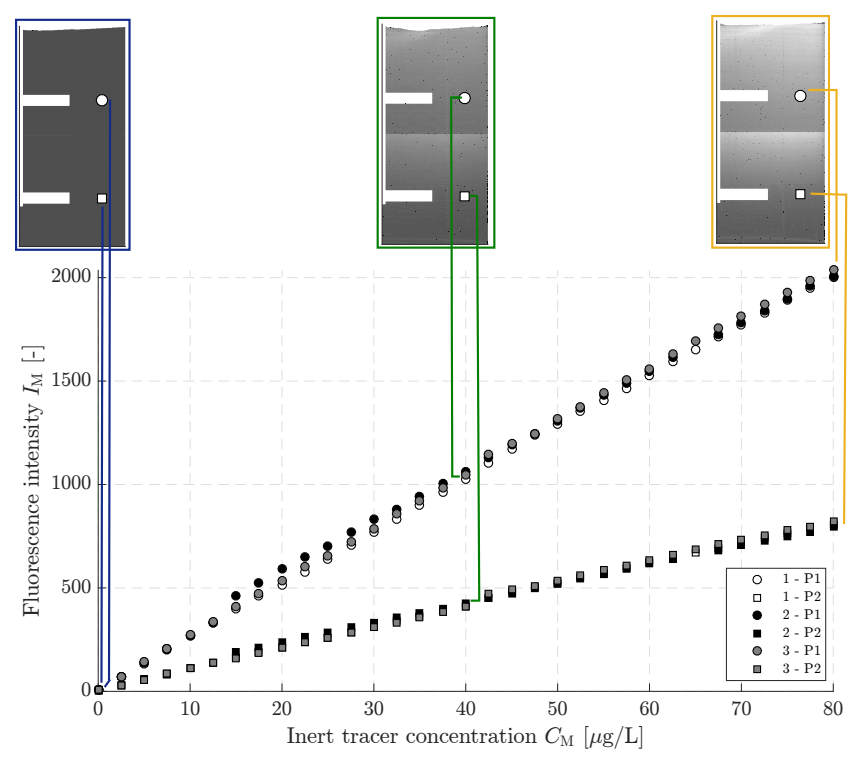

Figure A.1: PLIF calibration for $C_{\mathrm{M}}(r, z, t)$ measurements: sequence of intensity images and reproducibility between three runs $(1 \rightarrow 3)$ of calibration curves for two specified pixels (P1 and P2)

$80 \mu \mathrm{g} / \mathrm{L}$ for all runs. For larger concentrations, the linear approximation of the Beer-Lambert law through a MacLaurin expansion (Eq.(6)) is no longer valid (Albani (2008), Lakowicz 
(2013)).

Moreover, it is worth underlining that the results presented here are only bi-dimensional. The PLIF measurements performed are however sufficiently representative of the conditions in the whole vessel regarding the agitation speed and the low viscosity fluid used in this work (Busciglio et al. (2015)).

\section{Appendix A.2. Mass transfer}

Before starting the experiments to measure the dissolved oxygen instantaneous concentration field $C_{\mathrm{O}_{2}}(r, z, t)$, the relation between the grayscale value and the dissolved oxygen concentration (Eq.(7)) for each pixel has been determined by a calibration procedure, during which the dissolved oxygen concentration was successively maintained at different known values in the liquid phase. Subsequently, three runs $(1 \rightarrow 3)$ were carried out to check the accuracy and reliability of the system calibration. The fluorescence intensity for each pixel is recorded for each run as a function of dissolved oxygen concentration. As an example, results plotted in Figure A.2 are relative to concentration measurements at two pixels $\mathrm{P} 1$ and $\mathrm{P} 2$, respectively located in the upper and lower parts of the tank. For all runs, an

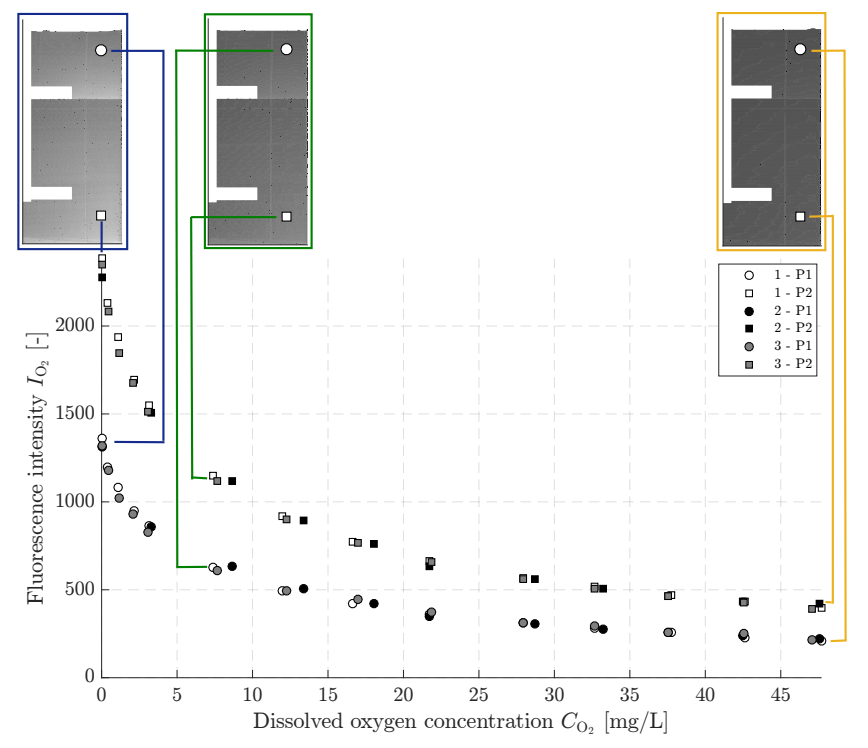

Figure A.2: PLIF calibration for $C_{\mathrm{O}_{2}}(r, z, t)$ measurements: reproducibility between three runs $(1 \rightarrow 3)$ of calibration curves for two specified pixels ( $\mathrm{P} 1$ and P2)

inverse proportionality relation, in agreement with Equation 7, has been observed for the whole range of dissolved oxygen concentration, which corresponds to liquid saturation values relative to a gas phase containing from 0 to $100 \%$ of oxygen. As for PLIF mixing data, the three sets of points show the satisfactory reproducibility of the PLIF measurements, which thus may be used to assess the transfer dynamics inside the stirred tank.

Moreover, as previously explained for the mixing results, these 2D-PLIF measurements are representative enough of the conditions in the whole vessel, but are subject to turbulence and experimental noise.

\section{Appendix B. Taylor macro- and micro-scales calculations}

The determination of $\Lambda_{i i}$ and $\lambda_{i i}$ from Equations 12 and 13 respectively is not straightforward.

The form of the autocorrelation function in Equation 12 is such that it generally decreases rapidly to its first zero-crossing, after which it may become negative and oscillates around zero. Moreover, the domain of the autocorrelation function from experimental data is finite, bounded by the tank wall. The longitudinal integral length-scales $\Lambda_{r r}$ and $\Lambda_{z z}$ are estimated by integrating the corresponding autocorrelation functions up to the first zero in a given direction (two possibilities for each: $\Lambda_{r r}$ right $\Lambda_{r r}$ or left $\Lambda_{r r}^{\leftarrow}$, and $\Lambda_{z z}-$ up $\Lambda_{z z}^{\uparrow}$ and down $\Lambda_{z z}^{\downarrow}$ ). Each pair is then weight-averaged according to the relative position of the domain limits. By way of illustration, the weight of $\Lambda_{r r}$ is going from $1(r=0)$ to $0(r=T / 2)$ as the effect of the tank wall is more and more important, and in return the weight of $\Lambda_{r r}^{\leftarrow}$ is going from $0(r=0)$ to $1(r=T / 2)$ as the effect of the impeller axis is less and less important. Besides, when the autocorrelation function presents no values close zero due to the tank limits, $\Lambda_{r r}$ or $\Lambda_{z z}$ is computed by fitting an exponential function (Hinze (1959), Bugay (1998)):

$$
\begin{aligned}
& R_{r r}=\exp \left(\frac{-d r}{\Lambda_{r r}}\right) \\
& R_{z z}=\exp \left(\frac{-d z}{\Lambda_{z z}}\right)
\end{aligned}
$$

The expression of the length-scale $\lambda_{r r}$ or $\lambda_{z z}$ in Equation 13 is manipulated and related to velocity derivatives (Hinze (1959), Jakobsen (2008)):

$$
\begin{aligned}
& \lambda_{r r}(r, z)=\sqrt{\frac{-2}{\partial_{r=0}^{2} R_{r r}(r+d r, z)}} \equiv \sqrt{\frac{2 \overline{u_{r}^{\prime 2}}}{\overline{\left(\frac{\partial u_{r}^{\prime}}{\partial r}\right)^{2}}}} \\
& \lambda_{z z}(r, z)=\sqrt{\frac{-2}{\partial_{z=0}^{2} R_{z z}(r, z+d z)}} \equiv \sqrt{\frac{2 \overline{u_{z}^{\prime 2}}}{\left.\overline{\frac{\partial u_{z}^{\prime}}{\partial z}}\right)^{2}}}
\end{aligned}
$$

\section{References}

Albani, J. R., 2008. Principles and applications of fluorescence spectroscopy. John Wiley \& Sons.

Alberini, F., Simmons, M. J. H., Ingram, A., Stitt, E. H., 2014. Use of an areal distribution of mixing intensity to describe blending of non-newtonian fluids in a kenics KM static mixer using PLIF. AIChE Journal 60 (1), 332-342.

Bałdyga, J., Pohorecki, R., 1995. Turbulent micromixing in chemical reactors a review. The Chemical Engineering Journal and the Biochemical Engineering Journal 58 (2), 183 - 195.

Banerjee, S., Lakehal, D., Fulgosi, M., 2004. Surface divergence models for scalar exchange between turbulent streams. International Journal of Multiphase Flow 30 (7-8), 963 - 977.

Bouwmans, I., Bakker, A., Van den Akker, H., 1997. Fluid flow blending liquids of differing viscosities and densities in stirred vessels. Chemical Engineering Research and Design 75 (8), 777 - 783.

Brown, D. A. R., Jones, P. N., Middleton, J. C., Papadopoulos, G., Arik, E. B., 2004. Experimental methods. John Wiley \& Sons, Inc., pp. 145-256.

Bugay, S., 1998. Analyse locale des échelles caractéristiques du mélange: Application de la technique P.I.V. aux cuves agitées. Ph.D. thesis, Institut National des Sciences Appliquées, Toulouse, France.

Burmester, S. S. H., Rielly, C. D., Edwards, M. F., 1992. The mixing of miscible liquids with large differences in density and viscosity. Springer Netherlands, Dordrecht, pp. 83-90. 
Busciglio, A., Grisafi, F., Scargiali, F., Brucato, A., 2014. Mixing dynamics in uncovered unbaffled stirred tanks. Chemical Engineering Journal 254, 210 219

Busciglio, A., Montante, G., Paglianti, A., 2015. Flow field and homogenization time assessment in continuously-fed stirred tanks. Chemical Engineering Research and Design 102, 42 - 56.

Cents, A., de Bruijn, F., Brilman, D., Versteeg, G., 2005. Validation of the danckwerts-plot technique by simultaneous chemical absorption of $\mathrm{CO}_{2}$ and physical desorption of $\mathrm{O}_{2}$. Chemical Engineering Science 60 (21), $5809-$ 5818

Chu, C. R., Jirka, G. H., 1992. Turbulent gas flux measurements below the airwater interface of a grid-stirred tank. International Journal of Heat and Mass Transfer 35 (8), 1957 - 1968.

Chung, K., Barigou, M., Simmons, M., 2007. Reconstruction of 3-D flow field inside miniature stirred vessels using a 2-D PIV technique. Chemical Engineering Research and Design 85 (5), 560 - 567

Coroneo, M., Montante, G., Paglianti, A., Magelli, F., 2011. CFD prediction of fluid flow and mixing in stirred tanks: Numerical issues about the RANS simulations. Computers \& Chemical Engineering 35 (10), 1959 - 1968.

Crimaldi, J. P., 2008. Planar laser-induced fluorescence in aqueous flows. Experiments in Fluids 44 (6), 851-863

Danckwerts, P. V., 1951. Significance of liquid-film coefficients in gas absorption. Industrial \& Engineering Chemistry 43 (6), 1460-1467.

Danckwerts, P. V., 1952. The definition and measurement of some characteristics of mixtures. Applied Scientific Research, Section A 3 (4), 279-296.

Delafosse, A., Collignon, M.-L., Calvo, S., Delvigne, F., Crine, M., Thonart, P., Toye, D., 2014. CFD-based compartment model for description of mixing in bioreactors. Chemical Engineering Science 106, 76 - 85.

Delvigne, F., Lejeune, A., Destain, J., Thonart, P., 2006. Modelling of the substrate heterogeneities experienced by a limited microbial population in scaledown and in large-scale bioreactors. Chemical Engineering Journal 120 (3), $157-167$

Derksen, J., Doelman, M., Van den Akker, H., 1999. Three-dimensional LDA measurements in the impeller region of a turbulently stirred tank. Experiments in Fluids 27 (6), 522-532.

Enfors, S.-O., Jahic, M., Rozkov, A., Xu, B., Hecker, M., Jügen, B., Krüger, E., Schweder, T., Hamer, G., O’Beirne, D., Noisommit-Rizzi, N., Reuss, M., Boone, L., Hewitt, C., McFarlane, C., Nienow, A., Kovacs, T., Trägårdh, C., Fuchs, L., Revstedt, J., Friberg, P., Hjertager, B., Blomsten, G., Skogman, H., Hjort, S., Hoeks, F., Lin, H.-Y., Neubauer, P., van der Lans, R., Luyben, K., Vrabel, P., Manelius, A., 2001. Physiological responses to mixing in large scale bioreactors. Journal of Biotechnology 85 (2), 175 - 185.

Escudié, R., Liné, A., 2003. Experimental analysis of hydrodynamics in a radially agitated tank. AIChE Journal 49 (3), 585-603.

Escudié, R., Liné, A., 2006. Analysis of turbulence anisotropy in a mixing tank. Chemical Engineering Science 61 (9), 2771 - 2779

Falkenroth, A., Degreif, K., Jähne, B., 2007. Visualisation of oxygen concentration fields in the mass boundary layer by fluorescence quenching. Springer Berlin Heidelberg, Berlin, Heidelberg, pp. 59-72.

Fall, A., Lecoq, O., David, R., 2001. Characterization of mixing in a stirred tank by planar laser induced fluorescence (P.L.I.F.). Chemical Engineering Research and Design 79 (8), 876 - 882

Fortescue, G., Pearson, J., 1967. On gas absorption into a turbulent liquid. Chemical Engineering Science 22 (9), 1163 - 1176

Gabriele, A., Nienow, A., Simmons, M., 2009. Use of angle resolved PIV to estimate local specific energy dissipation rates for up- and down-pumping pitched blade agitators in a stirred tank. Chemical Engineering Science 64 (1), $126-143$.

Galletti, C., Brunazzi, E., Pintus, S., Paglianti, A., Yianneskis, M., 2004. Triple products and turbulence states in a radially stirred tank with 3-D laser anemometry. Chemical Engineering Research and Design 82 (9), 1214 1228 .

Garcia-Ochoa, F., Gomez, E., 2009. Bioreactor scale-up and oxygen transfer rate in microbial processes: An overview. Biotechnology Advances 27 (2), $153-176$.

Godoy-Silva, R., Berdugo, C., Chalmers, J. J., Flickinger, M. C., 2009. Aeration, Mixing, and Hydrodynamics, Animal Cell Bioreactors. John Wiley \& Sons, Inc.

Gogate, P. R., Pandit, A. B., 1999. Mixing of miscible liquids with density differences: Effect of volume and density of the tracer fluid. The Canadian Journal of Chemical Engineering 77 (5), 988-996.
Guillard, F., Trägårdh, C., Fuchs, L., 2000. A study on the instability of coherent mixing structures in a continuously stirred tank. Chemical Engineering Science 55 (23), 5657 - 5670 .

Hamborg, E. S., Kersten, S. R., Versteeg, G. F., 2010. Absorption and desorption mass transfer rates in non-reactive systems. Chemical Engineering Journal 161 (1-2), 191 - 195.

Hartmann, H., Derksen, J. J., Van den Akker, H. E. A., 2006. Mixing times in a turbulent stirred tank by means of LES. AIChE Journal 52 (11), 3696-3706.

Herlina, Jirka, G. H., 2004. Application of LIF to investigate gas transfer near the air-water interface in a grid-stirred tank. Experiments in Fluids 37 (3), 341-349.

Herlina, H., Wissink, J. G., 2014. Direct numerical simulation of turbulent scalar transport across a flat surface. Journal of Fluid Mechanics 744, 217 249

Hinze, J., 1959. Turbulence: An Introduction to Its Mechanism and Theory. McGraw-Hill series in Mechanical Engineering. McGraw-Hill.

Houcine, I., Vivier, H., Plasari, E., David, R., Villermaux, J., 1996. Planar laserinduced fluorescence technique for measurements of concentration fields in continuous stirred tank reactors. Experiments in Fluids 22 (2), 95-102.

Jakobsen, H. A., 2008. Single Phase Flow. Springer Berlin Heidelberg, Berlin, Heidelberg, pp. 3-185.

Janzen, J. G., Herlina, H., Jirka, G. H., Schulz, H. E., Gulliver, J. S., 2010. Estimation of mass transfer velocity based on measured turbulence parameters. AIChE Journal 56 (8), 2005-2017.

Jimenez, M., Dietrich, N., Cockx, A., Hébrard, G., 2013. Experimental study of $\mathrm{O}_{2}$ diffusion coefficient measurement at a planar gas-liquid interface by planar laser-induced fluorescence with inhibition. AIChE Journal 59 (1), 325333.

Jimenez, M., Dietrich, N., Grace, J. R., Hébrard, G., 2014. Oxygen mass transfer and hydrodynamic behaviour in wastewater: Determination of local impact of surfactants by visualization techniques. Water Research 58, 111 121

Kasat, G. R., Pandit, A. B., 2004. Mixing time studies in multiple impeller agitated reactors. The Canadian Journal of Chemical Engineering 82 (5), 892-904.

Khan, F., Rielly, C., Hargrave, G., 2004. A multi-block approach to obtain angle-resolved PIV measurements of the mean flow and turbulence fields in a stirred vessel. Chemical Engineering \& Technology 27 (3), 264-269.

Kukukova, A., Aubin, J., Kresta, S. M., 2009. A new definition of mixing and segregation: Three dimensions of a key process variable. Chemical Engineering Research and Design 87 (4), 633 - 647, 13th European Conference on Mixing: New developments towards more efficient and sustainable operations

Kukukova, A., Aubin, J., Kresta, S. M., 2011. Measuring the scale of segregation in mixing data. The Canadian Journal of Chemical Engineering 89 (5), 1122-1138.

Kukuková, A., Noël, B., Kresta, S. M., Aubin, J., 2008. Impact of sampling method and scale on the measurement of mixing and the coefficient of variance. AIChE Journal 54 (12), 3068-3083.

Lakowicz, J. R., 2013. Principles of fluorescence spectroscopy. Springer Science \& Business Media.

Lamont, J. C., Scott, D. S., 1970. An eddy cell model of mass transfer into the surface of a turbulent liquid. AIChE Journal 16 (4), 513-519.

Larsson, G., Törnkvist, M., Wernersson, E. S., Trägårdh, C., Noorman, H., Enfors, S. O., 1996. Substrate gradients in bioreactors: origin and consequences. Bioprocess Engineering 14 (6), 281-289.

Liné, A., 2016. Eigenvalue spectrum versus energy density spectrum in a mixing tank. Chemical Engineering Research and Design 108, 13 - 22.

Liné, A., Gabelle, J.-C., Morchain, J., Anne-Archard, D., Augier, F., 2013. On POD analysis of PIV measurements applied to mixing in a stirred vessel with a shear thinning fluid. Chemical Engineering Research and Design 91 (11), 2073 - 2083.

Linek, V., Vacek, V., Beneš, P., 1987. A critical review and experimental verification of the correct use of the dynamic method for the determination of oxygen transfer in aerated agitated vessels to water, electrolyte solutions and viscous liquids. The Chemical Engineering Journal 34 (1), 11 - 34.

Martín, M., Montes, F. J., Galán, M. A., 2008. On the contribution of the scales of mixing to the oxygen transfer in stirred tanks. Chemical Engineering Journal 145 (2), $232-241$

Mathpati, C. S., Tabib, M. V., Deshpande, S. S., Joshi, J. B., 2009. Dynamics of flow structures and transport phenomena, 2. Relationship with design objec- 
tives and design optimization. Industrial \& Engineering Chemistry Research 48 (17), 8285-8311.

McCready, M. J., Vassiliadou, E., Hanratty, T. J., 1986. Computer simulation of turbulent mass transfer at a mobile interface. AIChE Journal 32 (7), 11081115 .

Micheletti, M., Baldi, S., Yeoh, S., Ducci, A., Papadakis, G., Lee, K., Yianneskis, M., 2004. On spatial and temporal variations and estimates of energy dissipation in stirred reactors. Chemical Engineering Research and Design 82 (9), 1188 - 1198.

Montante, G., Coroneo, M., Paglianti, A., 2016. Blending of miscible liquids with different densities and viscosities in static mixers. Chemical Engineering Science 141, $250-260$.

Morchain, J., Gabelle, J., Cockx, A., 2014. A coupled population balance model and CFD approach for the simulation of mixing issues in lab-scale and industrial bioreactors. AIChE Journal 60 (1), 27-40.

Nienow, A., 1997. On impeller circulation and mixing effectiveness in the turbulent flow regime. Chemical Engineering Science 52 (15), 2557 - 2565.

Rielly, C., Smith, D., Lindley, J., Niranjan, K., Phillips, V., 1994. Mixing processes for agricultural and food materials: Part 4, Assessment and monitoring of mixing systems. Journal of Agricultural Engineering Research 59 (1), $1-18$.

Scargiali, F., Busciglio, A., Grisafi, F., Brucato, A., 2010. Simplified dynamic pressure method for measurement in aerated bioreactors. Biochemical Engineering Journal 49 (2), 165 - 172.

Taylor, G. I., 1935. Statistical theory of turbulence. Proceedings of the Royal Society of London A: Mathematical, Physical and Engineering Sciences 151 (873), 421-444.

Tsumori, H., Sugihara, Y., 2007. Lengthscales of motions that control air-water gas transfer in grid-stirred turbulence. Journal of Marine Systems 66 (1-4), $6-18$.

Van't Riet, K., 1979. Review of measuring methods and results in nonviscous gas-liquid mass transfer in stirred vessels. Industrial \& Engineering Chemistry Process Design and Development 18 (3), 357-364

Variano, E. A., Cowen, E. A., 2013. Turbulent transport of a high-schmidtnumber scalar near an air-water interface. Journal of Fluid Mechanics 731, 259-287.

Wernersson, E. S., Trägårdh, C., 1999. Scale-up of rushton turbine-agitated tanks. Chemical Engineering Science 54 (19), 4245 - 4256.

Woodrow, P. T., Duke, S. R., 2001. Laser-induced fluorescence studies of oxygen transfer across unsheared flat and wavy air-water interfaces. Industrial \& Engineering Chemistry Research 40 (8), 1985-1995. 